\title{
Dysregulation of brain and choroid plexus cell types in severe COVID-19
}

https://doi.org/10.1038/s41586-021-03710-0

Received: 20 October 2020

Accepted: 7 June 2021

Published online: 21 June 2021

Check for updates

\author{
Andrew C. Yang ${ }^{1,2,3,11}$, Fabian Kern ${ }^{4,11}$, Patricia M. Losada ${ }^{3}$, Maayan R. Agam ${ }^{3}$, \\ Christina A. Maat ${ }^{3}$, Georges P. Schmartz ${ }^{4}$, Tobias Fehlmann ${ }^{4}$, Julian A. Stein ${ }^{5}$, \\ Nicholas Schaum ${ }^{3}$, Davis P. Lee ${ }^{3}$, Kruti Calcuttawala ${ }^{3}$, Ryan T. Vest ${ }^{3}$, Daniela Berdnik ${ }^{3}$, \\ Nannan Lu ${ }^{3}$, Oliver Hahn ${ }^{3}$, David Gate ${ }^{3}$, M. Windy McNerney ${ }^{6}$, Divya Channappa ${ }^{3}$, \\ Inma Cobos ${ }^{3,7}$, Nicole Ludwig ${ }^{8}$, Walter J. Schulz-Schaeffer ${ }^{5}$, Andreas Keller ${ }^{3,4,12}$ \& \\ Tony Wyss-Coray ${ }^{2,3,9,10,12 \bowtie ~}$
}

\begin{abstract}
Although SARS-CoV-2 primarily targets the respiratory system, patients with and survivors of COVID-19 can suffer neurological symptoms ${ }^{1-3}$. However, an unbiased understanding of the cellular and molecular processes that are affected in the brains of patients with COVID-19 is missing. Here we profile 65,309 single-nucleus transcriptomes from 30 frontal cortex and choroid plexus samples across 14 control individuals (including 1 patient with terminal influenza) and 8 patients with COVID-19. Although our systematic analysis yields no molecular traces of SARS-CoV-2 in the brain, we observe broad cellular perturbations indicating that barrier cells of the choroid plexus sense and relay peripheral inflammation into the brain and show that peripheral $\mathrm{T}$ cells infiltrate the parenchyma. We discover microglia and astrocyte subpopulations associated with COVID-19 that share features with pathological cell states that have previously been reported in human neurodegenerative disease $\mathrm{e}^{4-6}$. Synaptic signalling of upper-layer excitatory neurons-which are evolutionarily expanded in humans ${ }^{7}$ and linked to cognitive function ${ }^{8}$-is preferentially affected in COVID-19. Across cell types, perturbations associated with COVID-19 overlap with those found in chronic brain disorders and reside in genetic variants associated with cognition, schizophrenia and depression. Our findings and public dataset provide a molecular framework to understand current observations of COVID-19-related neurological disease, and any such disease that may emerge at a later date.
\end{abstract}

Patients with COVID-19 can suffer neurological and psychiatric symptoms that range from loss of smell and headache to encephalitis and stroke $^{1-3,9-11}$. These symptoms are more prevalent in patients who are hospitalized ${ }^{1,12,13}$ and may persist as 'long COVID', which consists of 'brain fog', difficulty in concentrating and fatigue $\mathrm{e}^{14,15}$.

Cellular and molecular approaches are required to understand the neurological changes that may contribute to symptoms reported in patients with COVID-19. Neuropathology may arise from direct virus neuroinvasion or indirectly from peripheral infection and its attendant immune response ${ }^{16}$. Thus, much attention has been paid to whether SARS-CoV-2 can be detected in the brain, which has yielded inconsistent results ${ }^{9,17-21}$. Critically, a comprehensive assessment across specific cell types in the brain affected by severe COVID-19 is missing. This is in part because the high-quality, fresh-frozen human brain tissue from patients with COVID-19 needed for single-cell transcriptomic studies is largely inaccessible, and methods to isolate human brain barrier cells have only recently emerged ${ }^{22,23}$.
Here we characterized the transcriptomes of 65,309 nuclei isolated from the brains of 14 control individuals and 8 patients with COVID19 (Fig. 1a, Supplementary Table 1). We created an interactive data browser (https://twc-stanford.shinyapps.io/scRNA_Brain_COVID19) to provide researchers with a comprehensive resource to further investigate the molecular mechanisms of the effects of SARS-CoV-2 on the brain.

\section{Cortex and choroid plexus cell types}

We generated 38,217 single-nucleus gene-expression profiles from the medial frontal cortex ( 8 control individuals and 8 patients with COVID19) and detected a median of 1,918 genes per nucleus, consistent with recent studies ${ }^{5,8,24,25}$ (Fig. 1b, Extended Data Fig. 1a). Our sample sizes were similar to or greater than those reported in previous COVID-19 or brain single-nucleus RNA-sequencing (snRNA-seq) studies ${ }^{24-26}$. The samples in the control and COVID-19 groups were from individuals

${ }^{1}$ Department of Bioengineering, Stanford University School of Medicine, Stanford, CA, USA. ${ }^{2} \mathrm{ChEM}-\mathrm{H}$, Stanford University, Stanford, CA, USA. ${ }^{3}$ Department of Neurology and Neurological Sciences, Stanford University School of Medicine, Stanford, CA, USA. ${ }^{4}$ Chair for Clinical Bioinformatics, Saarland University, Saarbrücken, Germany. ${ }^{5}$ Institute for Neuropathology, Saarland University Hospital and Medical Faculty of Saarland University, Homburg, Germany. ${ }^{6}$ Department of Psychiatry, Stanford University School of Medicine, Stanford, CA, USA. ${ }^{7}$ Department of Pathology, Stanford University School of Medicine, Stanford, CA, USA. ${ }^{8}$ Department of Human Genetics, Saarland University, Homburg, Germany. ${ }^{9}$ Wu Tsai Neurosciences Institute, Stanford

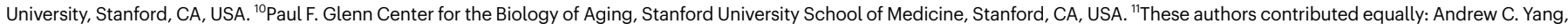
Fabian Kern. ${ }^{12}$ These authors jointly supervised this work: Andreas Keller, Tony Wyss-Coray. ${ }^{\bowtie}$ e-mail: andreas.keller@ccb.uni-saarland.de; twc@stanford.edu 
a

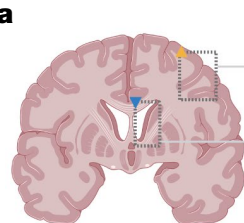

30 samples (22 patients)

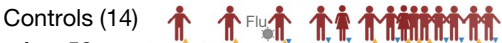

Age $50 \ldots-05$

covID-19 (8)

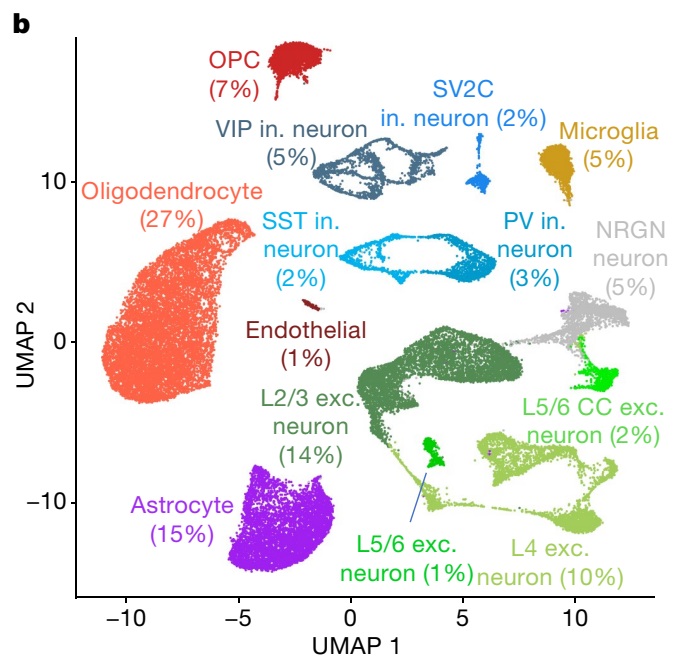

e

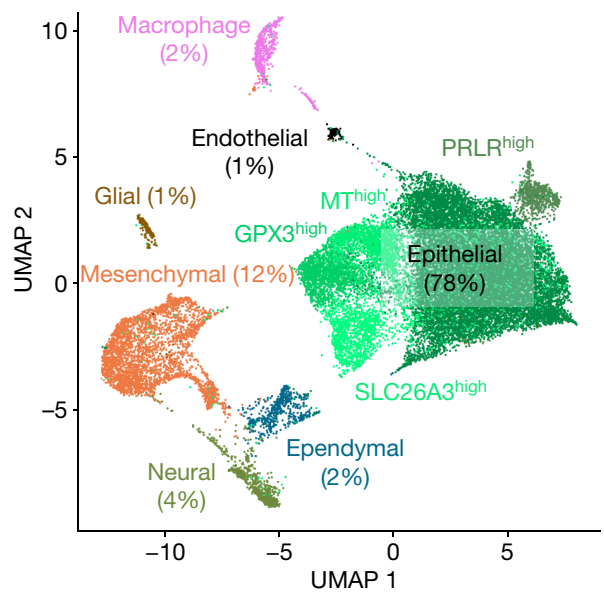

Fig. 1 Overview of diverse brain and choroid plexus cell types captured from post-mortem tissue from patients with COVID-19. a, Study design. Coloured triangles denote the brain regions that were studied for each patient. IHC, immunohistochemistry. b, Uniform manifold approximation and projection (UMAP) of 38,217 nuclei from the medial frontal cortex of 8 control individuals (including 1 patient with influenza) and 8 patients with COVID-19. As in previous reports ${ }^{5,25,46}$, the 'endothelial' cluster also exhibits vascular mural cell markers and perivascular cells (perivascular fibroblast-like cells and perivascular macrophages) are not efficiently captured. exc., excitatory; in., inhibitory; OPC, oligodendrocyte precursor cell.c, Examples of DEGs in COVID-19 ( $n=7$ control individuals (without viral infection); $n=8$ patients with COVID-19; MAST with default settings): excitatory neurons (exc. n.), inhibitory neurons (in. n.), astrocytes (ast.), oligodendrocytes (oli.), OPCs, and microglia and macrophages (mic./mac.). DEGs defined as log-transformed fold change $>0.25$ (absolute value) and adjusted $P$ value $<0.05$ (Bonferroni

between 55 and 91 years of age and matched for tissue dissection area, tissue and RNA quality (Extended Data Fig. 1b, c, Supplementary Table 1). The cause of death for nearly all patients with COVID-19 c

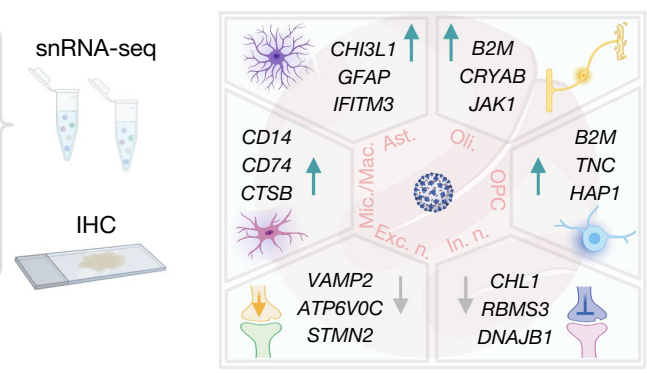

d

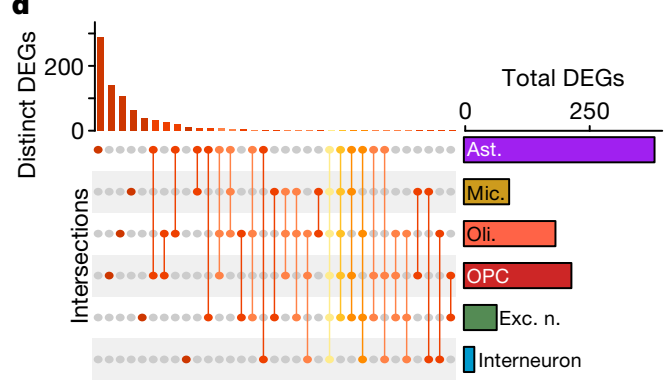

$\mathbf{f}$

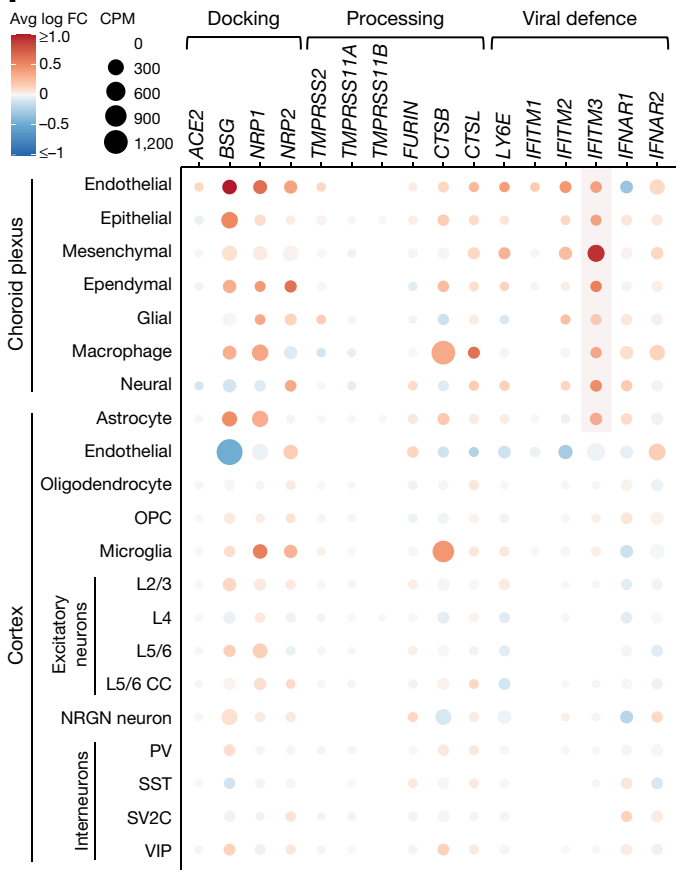

correction).d, Cell-type specificity of cortical DEGs. UpSet plot showing a matrix layout of DEGs shared across and specific to each cell type. Each matrix column represents either DEGs specific to a cell type (single circle with no vertical lines) or DEGs shared between cell types, with the vertical line indicating the cell types that share that given DEG. Top, bar graph displays the number of DEGs in each combination of cell types. Right, bar graph displays the total number of DEGs for a given cell type. e, UMAP of 27,092 nuclei from the lateral choroid plexus of 14 individuals $(n=7$ control individuals (including 1 patient with influenza); $n=7$ patients with COVID-19; MAST with default settings).f, Expression profiles (counts per million reads mapped (CPM)) (circle size) and differential expression in patients with COVID-19 (average log-transformed fold change (avg log FC)) (colour) for genes relevant to SARS-CoV-2 entry into the brain ${ }^{16}$. The highlighted region indicates the consistent upregulation of the antiviral defence gene IFITM 3 in choroid and glia limitans brain-barrier cells.

or influenza was interstitial pneumonia after more than two weeks of mechanical ventilation. Samples were not confounded by technical or batch artefacts (Extended Data Fig. 2). 
a

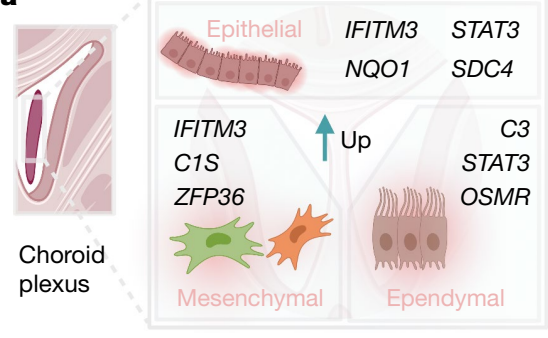

b
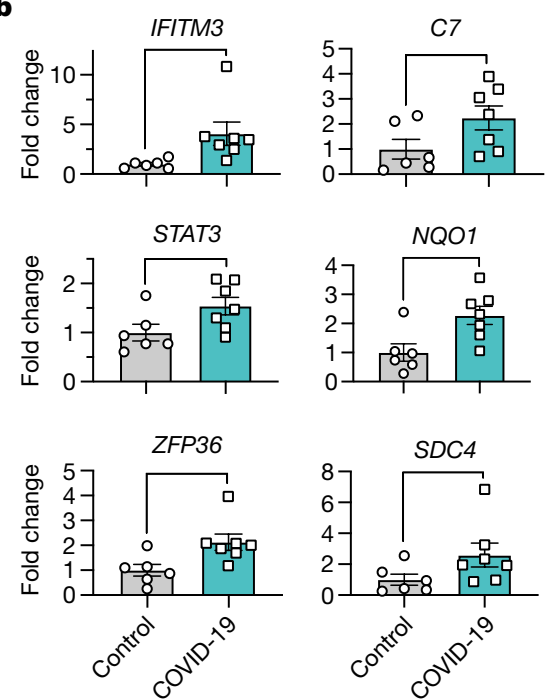

Fig. 2 | Brain-barrier inflammation in patients with COVID-19 does not require direct replicative infection. a, Examples of inflammation-related DEGs in the choroid plexus of patients with COVID-19 $(n=6$ control individuals (without viral infection); $n=7$ patients with COVID-19; MAST with default settings). DEGs defined as log-transformed fold change $>0.25$ (absolute value) and adjusted $P$ value $<0.05$ (Bonferroni correction). b, Validation of predicted choroid plexus DEGs by RT-qPCR ( $n=6$ control individuals (without viral infection), $n=7$ patients with COVID-19; two-sided Mann-Whitney $t$-test; mean \pm s.e.m.). Genes chosen for validation are either immediately related to SARS-CoV-2 (IFITM3) or genes with log-transformed fold changes similar to those of IFITM3 (NQO1), to assess the robustness of snRNA-seq thresholds. $P$ values $P=0.0023$ (IFITM3), 0.0484 (C7), 0.0350 (STAT3), 0.0140 (NQO1), 0.0082 (ZFP36) and 0.0734 (SDC4).c, snRNA-seq (left) or bulk RNA-seq (right) of choroid plexus and cortex from control individuals or patients with COVID-19 (no reads). snRNA-seq, $n=7$ control, $n=7$ COVID-19 (choroid plexus);

Our unsupervised clustering of nuclear transcriptomes yielded 14 cell types, including subtypes of excitatory neurons and interneurons that express previously established marker genes (Extended Data Fig. 3) and proportional to previous snRNA-seq data from adult human cortex ${ }^{5,8,24,25}$ (Extended Data Figs. 1-3, Supplementary Table 2).

We collapsed nuclei into 6 broad cell types, and identified 786 unique differentially expressed genes (DEGs) that implicated all major cell types (Fig. 1c, Extended Data Fig. 4). DEGs strongly correlated with alternative pseudobulk methods (but with greater statistical power (Extended Data Fig. 5)); and showed no significant overlap with genes affected by post-mortem delay to autopsy ${ }^{27}$ (Extended Data Fig. 6). Broadly, the strongest effects were seen in astrocytes and other glia, marked by inflammatory and dysregulated homeostatic pathways (Fig. 1c, Extended Data Fig. 4). The majority of DEGs were perturbed in only a single cell type (about $80 \%$ ) (Fig.1d).Several DEGs upregulated in one cell type were downregulated in others (Supplementary Tables 3,5). Overall, these data demonstrate that all major brain parenchymal cell types are affected in COVID-19.

Recent reports have found SARS-CoV-2 infection of cultured choroid plexus organoids ${ }^{20,21}$ but to our knowledge no snRNA-seq study exists on
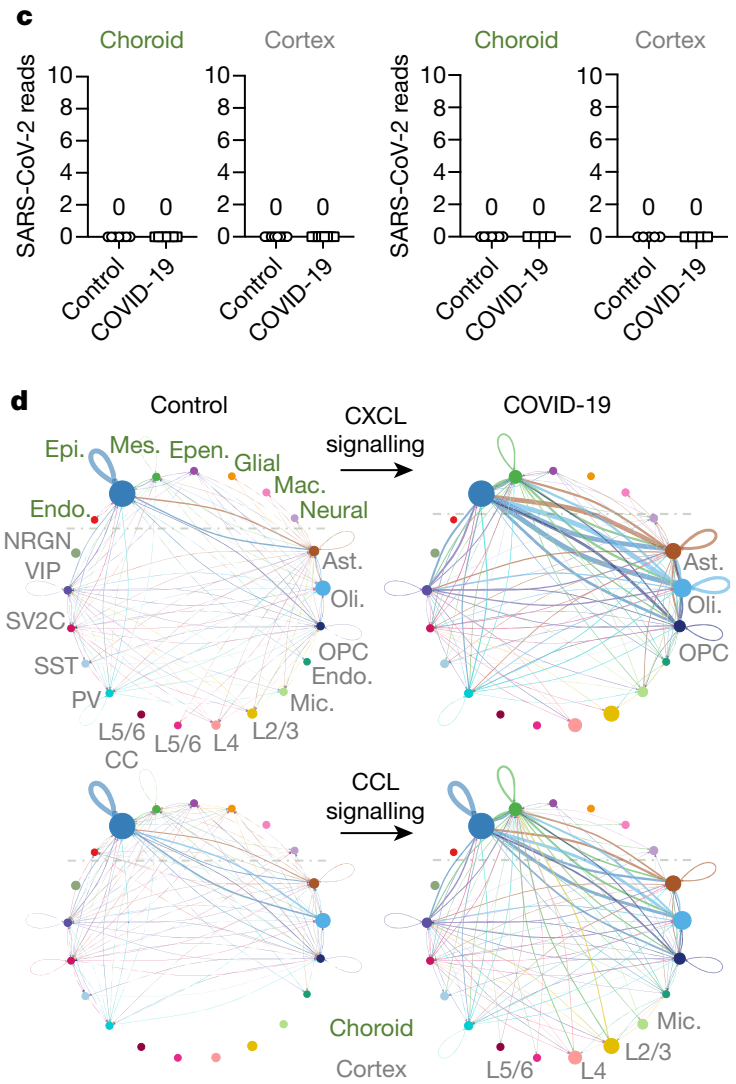

$n=7$ control, $n=7$ COVID-19 (cortex). Bulk RNA-seq (after viral RNA isolation): $n=7$ control, $n=4$ COVID-19 (choroid plexus); $n=5$ control, $n=4$ COVID-19 (cortex).d, Circle plot showing the number of statistically significant intercellular signalling interactions for the CXCL and CCL pathway family of molecules in control individuals compared to patients with COVID-19 (permutation test, CellChat ${ }^{32} ; n=8$ control individuals (including patients with influenza); $n=8$ patients with COVID-19 (cortex); and $n=7$ control individuals (including patients with influenza); $n=7$ patients with COVID-19 (choroid plexus)). Each circle (colour) represents one cell type; edges connecting circles represent significant intercellular signalling inferred between those cell types. Circles and edges are normalized to the number of cells for a given cell type and inferred strength of signalling, respectively. Cell types labelled on the right correspond to signalling pathways increased in COVID-19. Endo., endothelial; epen., ependymal; epi., epithelial; mes., mesenchymal.

the human choroid plexus, in health or disease ${ }^{22}$. We thus developed a method (Methods) that yielded 27,092 nuclei across 7 major epithelial, mesenchymal, immune, ependymal and glial cell types (7 control individuals and 7 patients with COVID-19) (Fig. 1e, Extended Data Fig. 3b, Supplementary Table 4). With capture of both brain parenchymal and barrier cell types, we assessed the expression and disease perturbation of genes related to SARS-CoV-2 entry, docking and defence ${ }^{16}$. Similar to brain vascular cells, choroid barrier cells robustly expressed several genes that are relevant to SARS-CoV-2 brain entry (Fig. 1f, Extended Data Fig. 7). We observed a broad upregulation of the antiviral defence gene IFITM3 across choroid and glia limitans barrier cells in patients with COVID-19, consistent with potential SARS-CoV-2 infection. IFITM3 serves as the first line of defence against viral infection ${ }^{28}$ and its upregulation is a marker of SARS-CoV-2 infection across public datasets ${ }^{29}$.

\section{Brain barriers relay inflammation}

We observed a broad upregulation of inflammatory genes across various interferon (IFITM3 and STAT3), complement (C1S, C3 and so on) and 

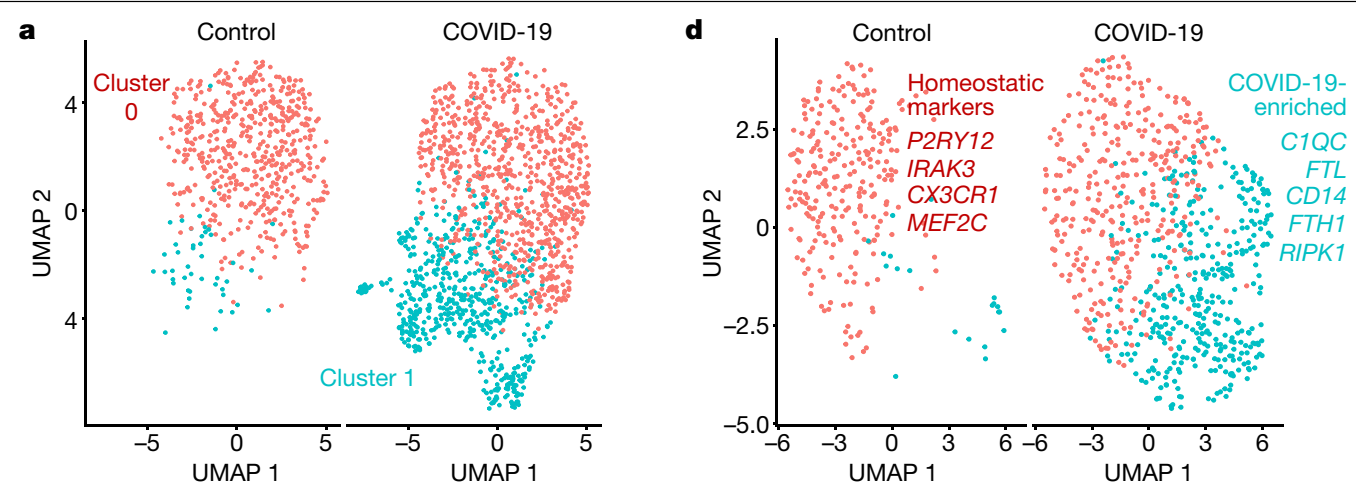

b
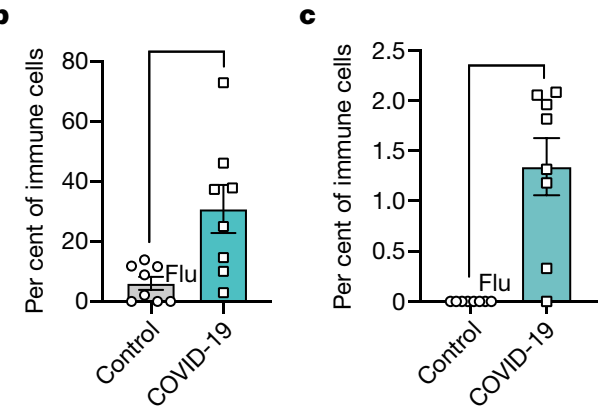

$\mathbf{g}$

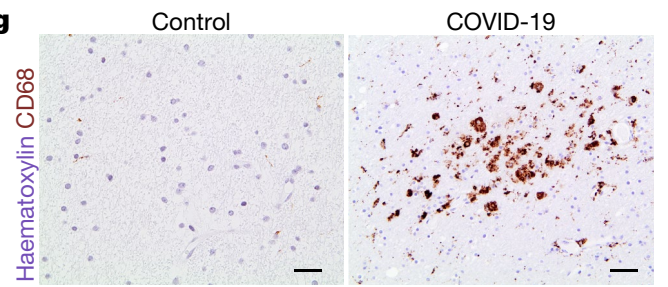

Fig. 3 | A neuroinflammatory COVID-19 milieu marked by diseaseassociated microglia. a, UMAP of immune cells captured in the human frontal cortex, split by control individuals (including a patient with influenza) $(n=8)$ (red) and patients with COVID-19 $(n=8)$ (light blue). Cells are coloured by cell-type subcluster (red cluster defined by homeostatic markers; light blue cluster defined by activation markers). b, Quantification of immune cell cluster 1 as a proportion of total immune cells ( $n=8$ control individuals (including a patient with influenza (circle marked as 'flu')); $n=8$ patients with COVID-19, two-sided Mann-Whitney $t$-test $P=0.0098$; mean \pm s.e.m.). c, As in b, but for T cells. $P=0.0003$. d, e, As in a, b, respectively, but for $\mathrm{MRC1}^{-}$parenchymal microglia. $P=0.0343$. Unlike macrophages, microglia express low levels of $M R C 1(\mathrm{CD} 206)^{34}$. Examples of genes that are upregulated in the microglial

related pathways across choroid plexus cell types (Fig. 2a). Quantitative PCR with reverse transcription (RT-qPCR) corroborated significant differential expression of tested inflammatory genes as well as other genes predicted to be upregulated by a similar magnitude in COVID-19 (for example, $N Q O 1$ and ZFP36) (Fig. 2b). Immunohistochemical staining also confirmed choroid plexus inflammation (Extended Data Fig. 8). Together, these data reveal substantial brain barrier inflammation in COVID-19 and validate the reliability of the DEGs that we identified in our snRNA-seq analysis.

Brain and choroid cell types express several SARS-CoV-2 entry genes (Fig. 1f, Extended Data Fig. 7) but claims of neuroinvasion in the literature have thus far been inconsistent ${ }^{9,17-20}$. To detect molecular evidence of SARS-CoV-2, we systematically performed four RNA-based and four antibody-based assays across our samples. RNA assays included searching for virus-specific reads (Methods) in our snRNA-seq dataset as well as in custom-generated bulk RNA-seq datasets with and without viral RNA enrichment. In no case did we detect SARS-CoV-2-specific RNA in the brain (Fig. 2c, Extended Data Fig. 9a). We confirmed this via

\section{e}
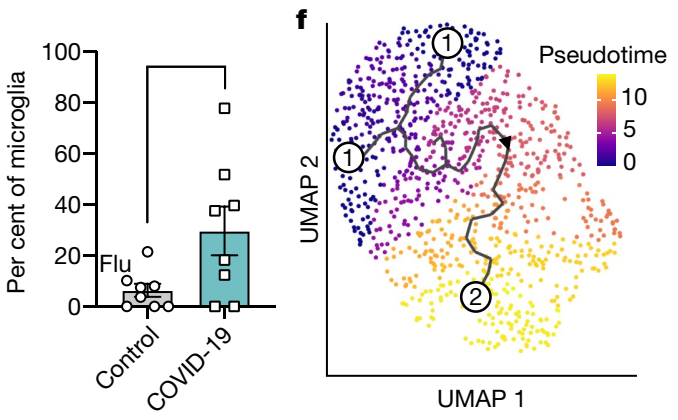

$\mathbf{h}$

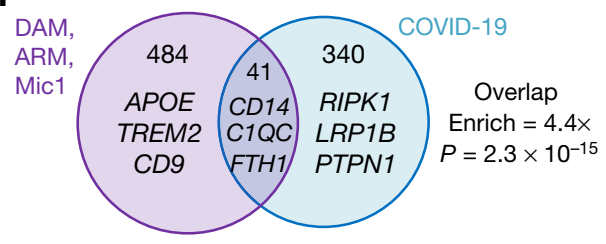

cluster associated with COVID-19 are shown in light blue.f, Pseudotime trajectory (Methods) indicated in graded purple (low) to yellow (high), plotting the emergence of the microglial cluster associated with COVID-19. Numbers indicate original source population (1) and the newly emerged population in COVID-19 (2).g, Immunohistochemical staining for the microglial activation marker CD68 (brown) in the frontal medial cortex of a patient with COVID-19, immediately adjacent to that used for snRNA-seq. Haematoxylin counterstain (blue).Scale bars, $20 \mu \mathrm{m}$. Immunohistochemical stains are representative of at least two independent experiments. h, Overlap (hypergeometric test) between marker genes of Alzheimer's-disease-associated microglia (DAM, ARM and Mic1) ${ }^{4-6}$ and genes that are upregulated in the microglial cluster associated with COVID-19.

qPCR using US Centers for Disease Control and Prevention Emergency Use Authorization primers against the $N 1$ and $N 2$ genes of the virus, again finding no enrichment in the brains of individuals with COVID-19 (Extended Data Fig. 9b). Some of the samples from the control individuals without viral infection have high cycle counts (between 37 and 40), which in previous work (without such controls) has been interpreted as evidence of neuroinvasion ${ }^{9,18}$. Finally, with the anti-SARS-CoV-2 spike (3A2) antibody used for immunohistochemistry (as in previous publications ${ }^{17,18}$ ), we observed signal across the barrier-forming cortical vasculature, meninges and choroid plexus (Extended Data Fig. 9c, d). Specific signal was retained across secondary detection methods (Extended Data Fig. 9e, Methods). However, no other antibody-including those also used in recent publications ${ }^{9,30}$-yielded specific signal over controls. Therefore, the $3 \mathrm{~A} 2$ antibody may bind a specific, but non-SARS-CoV-2, antigen.

The inflamed choroid plexus has previously been shown to send inflammatory signals into the brain, thereby activating parenchymal glia and impairing cognitive function ${ }^{31}$. To assess whether similar 


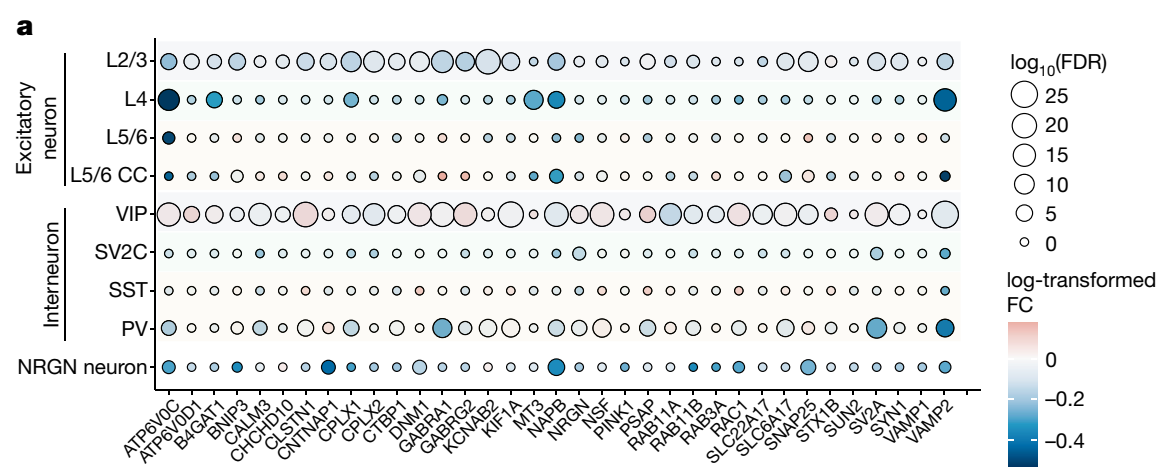

b

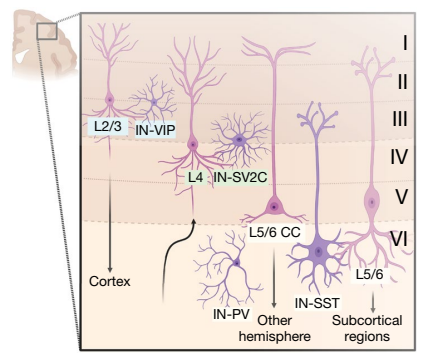

c

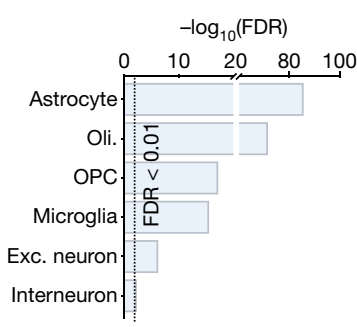

d

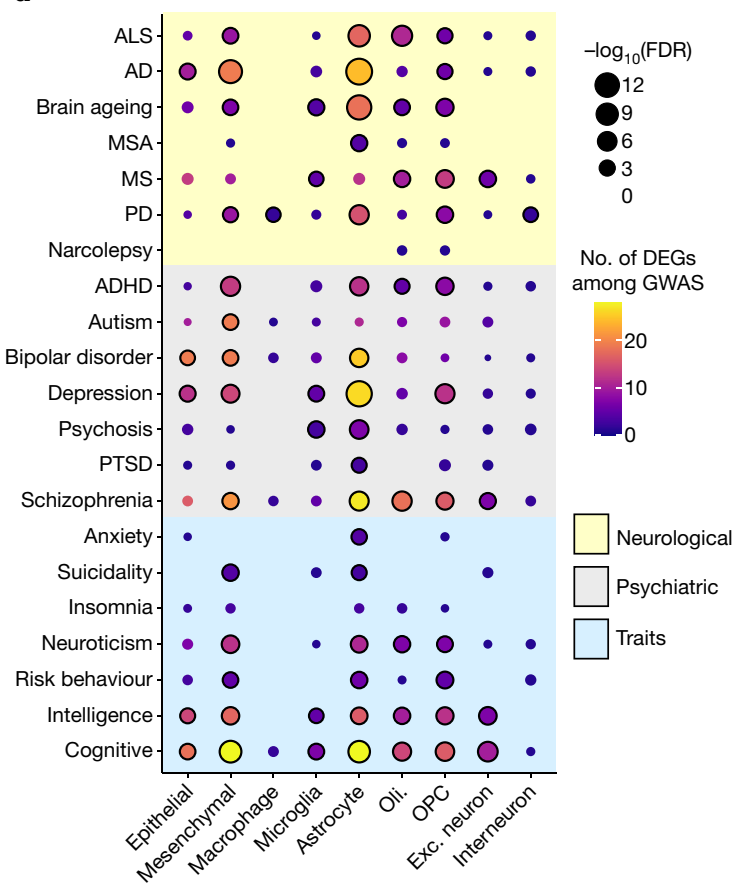

Fig. 4 | Molecular dysfunction in upper-layer neurons and links to longterm symptoms. a, Dot plot showing downregulation of synaptic vesicle components, especially in L2/3 excitatory neurons in patients with COVID-19 ( $n=7$ control individuals (without viral infection); $n=8$ patients with COVID-19; MAST with default settings). FC, fold change. b, Diagram of cortical neurons captured in this study that have known layer localization. Neuron labels are colour-coded by layer localization as shaded in a. Figure layout adapted with permission from ref. ${ }^{8} . \mathbf{c}$, Overlap between COVID-19 DEGs and those in chronic CNS diseases (Methods). Dotted line indicates statistical significance (adjusted
$P$ value $<0.05$, false-discovery rate (FDR) correction, cumulative hypergeometric test). d, Heat map showing the number of DEGs per cell type that overlap as GWAS risk variants across psychiatric and neurological diseases and traits from the GWAS catalogue (NHGRI-EBI) ${ }^{43}$. Significance of overlap is based on FDR-corrected cumulative hypergeometric $P$ values (BenjaminiHochberg correction) $<0.05$; MAST with default thresholds). AD, Alzheimer's disease; ADHD, attention deficit hyperactivity disorder; ALS, amyotrophic lateral sclerosis; MS, multiple sclerosis; MSA, multiple system atrophy; PD, Parkinson's disease; PTSD, post-traumatic stress disorder. pro-inflammatory relay mechanisms occur in the brains of patients with COVID-19, we performed cell-cell communication analysis ${ }^{32}$. We observed a strong increase in the choroid-to-cortex network across key inflammatory pathways, such as the CCL and CXCL family of chemokines from the choroid plexus epithelium to brain astrocytes, oligodendrocytes, microglia and layer (L) 2/3 and L4 excitatory neurons (Fig. 2d, Extended Data Fig. 10). Complement pathway signalling from the choroid plexus to brain microglia (the resident immune cells of the brain) was also predicted to increase in the brains of patients with COVID-19. Excessive complement signalling in microglia has previously been linked to premature neuronal synapse pruning in neurodegenerative disease ${ }^{33}$. Together, although we could not specifically detect virus RNA or protein in our brain samples, these results suggest that peripheral SARS-CoV-2 infection inflames brain-barrier cells such as those of the choroid plexus; and that this inflammation is then relayed into the brain parenchyma.

\section{Disease-associated microglia and astrocytes}

We thus sought to evaluate the immune landscape of the brain in individuals with COVID-19. We first analysed cortical immune cells, which contain mostly microglia but also lesser fractions of perivascular macrophages $\left(M R C 1^{+}\right.$, which encodes macrophage-specific mannose receptor $\left.C D 206^{34}\right)$ and T cells $\left(C D 247^{+}\right.$, which encodes the T cell receptor $\mathrm{CD} 3 \zeta$ protein). Our unsupervised clustering revealed the presence of a subpopulation of immune cells associated with COVID-19 (Fig. 3a), which was significantly enriched at both the per-nucleus and per-patient level (Fig. 3b). The emergence of disease-associated clusters reflects strong perturbations across the transcriptome. Similarly, although we did not find cortical T cells in any of our samples from control individuals (without viral infection or with terminal influenza), we detected them in all but one of the patients with COVID-19 (Fig. 3c). Aberrant $\mathrm{T}$ cell infiltration into the mouse brain has previously been 
reported to be sufficient to promote neuroinflammation and impair neurogenesis ${ }^{35}$.

To study microglia, we focused on the $M R C 1^{-} C D 247^{-}$immune cell subset to eliminate confounds from perivascular macrophages and T cells. Library quality was not affected upon restricting analyses to the $M R C 1^{-}$subset of microglia (Methods). We clustered $1,814 M R C 1^{-}$microglia, which revealed a distinct microglial subpopulation associated with COVID-19 (Fig. 3d) that was significant at both the per-nucleus and per-patient level (Fig. 3e). This subpopulation was marked by expression of microglial activation genes previously associated with human disease $e^{4,5}$, such as complement $C 1 Q C, C D 74, F T L$ and $F T H 1$, and downregulation of the homeostatic markers including $P 2 R Y 12$ (Fig. 3d, Supplementary Table 6). Trajectory analysis revealed that the microglia cluster associated with COVID-19 emerged from the parent homeostatic population (Fig. 3f), which further suggests that these microglia emerge in response to an increasingly inflamed central nervous system (CNS) environment. Our in situ staining confirmed the enriched presence of activated $\mathrm{CD} 68^{+}$parenchymal microglia in the brains of patients with COVID-19 as compared to those of control individuals (Fig. 3g, Extended Data Fig. 11); at times, these microglia form nodules that have previously been linked to viral encephalitis ${ }^{36}$ and myelin degeneration in ageing mice $\mathrm{e}^{37}$.

Microglial subclusters that are associated with disease have been identified for various neurodegenerative diseases ${ }^{4,5}$. A fraction of the genes enriched in the COVID-19-associated microglia cluster overlap $\left(P=2.3 \times 10^{-15}\right.$, hypergeometric test $)$ with those enriched in neurodegenerative-disease-associated microglia (Fig. $3 \mathrm{~h}$ ), including C1QC and CD14 (which mark microglia associated with Alzheimer's disease). Yet, several genes that have been implicated in neuroinflammation $^{38}$ (such as RIPK1) were seen specifically in microglial states associated with COVID-19. Our observations suggest that the microglial subpopulation enriched in patients with COVID-19 represents a distinct microglial state that shares features with-but is ultimately different from-microglial cell states that have previously been reported in human neurodegenerative disease.

In addition to abnormally activated microglia, we uncovered an astrocyte cluster associated with COVID-19 that is marked by established inflammation and astrogliosis genes (such as IFITM3 and GFAP) and upregulated expression of the secreted neurotoxic factor chitinase 3-like 1 (CHI3L1) ${ }^{39}$ (Extended Data Fig. 12a-c). Within this astrocyte cluster, we also observed significant dysregulation of genes that support neurotransmission and synaptic organization. By contrast, we did not observe any new subpopulations for oligodend rocyte lineage cells (Extended Data Fig.12d-g). Together, we identify the robust emergence of disease-associated microglia and astrocyte subpopulations with distinct transcriptional profiles in the brains of individuals with COVID-19.

\section{Links to long-term CNS dysfunction}

Given the predicted astrocytic impairments in supporting neurotransmission, we next sought to identify the neuronal subtypes that are most affected in COVID-19. Although we captured neurons from all cortical layers, we found gene-expression changes linked to synaptic deficits particularly in L2/3 excitatory neurons and L2/3-residing VIP interneurons ${ }^{40}$ (Fig. 4a, b). Specifically, the downregulation of synaptic genes that mediate neurotransmission (for example, VAMP2, SNAP25 and $A T P 6 V O C$ ) in L2/3 excitatory neurons alongside a concomitant upregulation in proximal VIP inhibitory neurons suggests dysfunction in upper-layer cortical circuitry. Such a pattern of dysfunction has previously been reported in an snRNA-seq study of autism and correlated with cognitive deficits ${ }^{8}$. L2/3 excitatory neurons are cortico-cortical projecting and already exhibit sparse action potential firing to generate a simple and reliable neural code for associative learning ${ }^{41}$. Thus, this neuronal population may be particularly sensitive to deficits in neurotransmission by COVID-19.
To investigate the potential pathologies that underlie reported neurological symptoms of long COVID, we analysed the intersection between COVID-19 DEGs across brain cell types with those that have previously been described in chronic CNS diseases, such as Alzheimer's disease ${ }^{5}$, multiple sclerosis ${ }^{26}$, Huntington's disease ${ }^{42}$ and autism spectrum disorder ${ }^{8}$. Although neuronal perturbations in COVID-19 were unique compared to those in chronic CNS diseases, the overlap in glial cells was particularly strong (Fig. 4c, Supplementary Table 7).

To further determine the enrichment of COVID-19 DEGs within genetic variants associated with complex traits and diseases in a cell-type-specific fashion, we obtained genome-wide association study (GWAS) summary statistics for neurological and psychiatric disorders and neurobehavioural traits ${ }^{43}$ (Supplementary Table 8 ). We found a strong enrichment of DEGs residing within GWAS hits of neurological disorders and traits, especially in cognition, schizophrenia and depression (Fig. 4d). Together, these data suggest that COVID-19 may partially recapitulate the pathological processes of various CNS diseases.

\section{Discussion}

Previous snRNA-seq studies have begun to elucidate the cell-type-specific perturbations and interactions involved in several CNS disorders ${ }^{5,8,25,26,42}$. Here, by combining sequencing of 65,309 nuclei in both the frontal cortex and choroid plexus, along with confirmatory immunohistochemistry and RT-qPCR, we reveal several major neuropathological mechanisms in severe COVID-19. However, there are limitations to consider. Most post-mortem brain tissue from individuals with COVID-19 is inadequately preserved or immediately fixed for safety and regulatory reasons, so there is a scarcity of high-quality tissue available for molecular studies. Also, although we did not detect SARS-CoV-2 in the choroid plexus or cortex, we cannot exclude the possibility of earlier neuroinvasion that had subsequently been cleared. Indeed, the mouse choroid plexus has recently been reported to express several SARS-CoV-2 entry factors ${ }^{22}$, which we corroborate in humans (Supplementary Discussion).

There is a precedent for acute viral infections causing long-term inflammation and dysfunction that predisposes individuals to neurodegenerative disease ${ }^{44,45}$, although not at the scale of the COVID-19 pandemic. It will be important to study how the molecular processes elucidated here contribute to the COVID-19 neurological symptoms and deficits of which we are aware now, and to those that may emerge in the years to come.

\section{Online content}

Any methods, additional references, Nature Research reporting summaries, source data, extended data, supplementary information, acknowledgements, peer review information; details of author contributions and competing interests; and statements of data and code availability are available at https://doi.org/10.1038/s41586-021-03710-0.

1. Mao, L. et al. Neurologic manifestations of hospitalized patients with coronavirus disease 2019 in Wuhan, China. JAMA Neurol. 77, 683-690 (2020).

2. Yang, X. et al. Clinical course and outcomes of critically ill patients with SARS-CoV-2 pneumonia in Wuhan, China: a single-centered, retrospective, observational study. Lancet Respir. Med. 8, 475-481 (2020).

3. Helms, J. et al. Neurologic features in severe SARS-CoV-2 infection. N. Engl. J. Med. 382, 2268-2270 (2020).

4. Keren-Shaul, H. et al. A unique microglia type associated with restricting development of Alzheimer's disease. Cell 169, 1276-1290 (2017).

5. Mathys, H. et al. Single-cell transcriptomic analysis of Alzheimer's disease. Nature $\mathbf{5 7 0}$, 332-337 (2019).

6. Sala Frigerio, C. et al. The major risk factors for Alzheimer's disease: age, sex, and genes modulate the microglia response to A 3 plaques. Cell Rep. 27, 1293-1306 (2019).

7. Gidon, A. et al. Dendritic action potentials and computation in human layer $2 / 3$ cortical neurons. Science 367, 83-87 (2020).

8. Velmeshev, D. et al. Single-cell genomics identifies cell type-specific molecular changes in autism. Science 364, 685-689 (2019). 
9. Matschke, J. et al. Neuropathology of patients with COVID-19 in Germany: a post-mortem case series. Lancet Neurol. 19, 919-929 (2020).

10. Varatharaj, A. et al. Neurological and neuropsychiatric complications of COVID-19 in 153 patients: a UK-wide surveillance study. Lancet Psychiatry 7, 875-882 (2020).

11. Ellul, M. A. et al. Neurological associations of COVID-19. Lancet Neurol. 19, 767-783 (2020).

12. Romero-Sánchez, C. M. et al. Neurologic manifestations in hospitalized patients with COVID-19: the ALBACOVID registry. Neurology 95, e1060-e1070 (2020)

13. Liotta, E. M. et al. Frequent neurologic manifestations and encephalopathy-associated morbidity in Covid-19 patients. Ann. Clin. Transl. Neurol. 7, 2221-2230 (2020).

14. Office for National Statistics. The prevalence of long COVID symptoms and COVID-19 complications, Office for National Statistics, https://www.ons.gov.uk/news/ statementsandletters/theprevalenceoflongcovidsymptomsandcovid19complications (2020).

15. Carfi, A., Bernabei, R. \& Landi, F. Persistent symptoms in patients after acute COVID-19. J. Am. Med. Assoc. 324, 603-605 (2020).

16. ladecola, C., Anrather, J. \& Kamel, H. Effects of COVID-19 on the nervous system. Cell 183, 16-27 (2020).

17. Cantuti-Castelvetri, L. et al. Neuropilin-1 facilitates SARS-CoV-2 cell entry and infectivity. Science 370, 856-860 (2020).

18. Meinhardt, J. et al. Olfactory transmucosal SARS-CoV-2 invasion as a port of central nervous system entry in individuals with COVID-19. Nat. Neurosci. 24, 168-175 (2021).

19. Song, E. et al. Neuroinvasion of SARS-CoV-2 in human and mouse brain. J. Exp. Med. 218, e20202135 (2021).

20. Jacob, F. et al. Human pluripotent stem cell-derived neural cells and brain organoids reveal SARS-CoV-2 neurotropism predominates in choroid plexus epithelium. Cell Stem Cell 27, 937-950 (2020).

21. Pellegrini, L. et al. SARS-CoV-2 infects the brain choroid plexus and disrupts the blood-CSF barrier in human brain organoids. Cell Stem Cell 27, 951-961 (2020).

22. Dani, N. et al. A cellular and spatial map of the choroid plexus across brain ventricles and ages. Cell 184, 3056-3074 (2021).

23. Yang, A. C. et al. A human brain vascular atlas reveals diverse cell mediators of Alzheimer's disease risk. Preprint at https://doi.org/10.1101/2021.04.26.441262 (2021).

24. Lake, B. B. et al. Integrative single-cell analysis of transcriptional and epigenetic states in the human adult brain. Nat. Biotechnol. 36, 70-80 (2018).

25. Grubman, A. et al. A single-cell atlas of entorhinal cortex from individuals with Alzheimer's disease reveals cell-type-specific gene expression regulation. Nat. Neurosci. 22, 2087-2097 (2019).

26. Jäkel, S. et al. Altered human oligodendrocyte heterogeneity in multiple sclerosis. Nature 566, 543-547 (2019).

27. Dachet, F. et al. Selective time-dependent changes in activity and cell-specific gene expression in human postmortem brain. Sci. Rep. 11, 6078 (2021).

28. Bailey, C. C., Zhong, G., Huang, I. C. \& Farzan, M. IFITM-family proteins: the cell's first line of antiviral defense. Annu. Rev. Virol. 1, 261-283 (2014).
29. Hachim, M. Y. et al. Interferon-induced transmembrane protein (IFITM3) is upregulated explicitly in SARS-CoV-2 infected lung epithelial cells. Front. Immunol. 11, 1372 (2020).

30. Rockx, B. et al. Comparative pathogenesis of COVID-19, MERS, and SARS in a nonhuman primate model. Science 368, 1012-1015 (2020).

31. Baruch, K. et al. Aging-induced type I interferon response at the choroid plexus negatively affects brain function. Science 346, 89-93 (2014).

32. Jin, S. et al. Inference and analysis of cell-cell communication using CellChat. Nat. Commun. 12, 1088 (2021).

33. Hong, S. et al. Complement and microglia mediate early synapse loss in Alzheimer mouse models. Science 352, 712-716 (2016).

34. Prinz, M., Erny, D. \& Hagemeyer, N. Ontogeny and homeostasis of CNS myeloid cells. Nat. Immunol. 18, 385-392 (2017).

35. Dulken, B. W. et al. Single-cell analysis reveals T cell infiltration in old neurogenic niches. Nature 571, 205-210 (2019).

36. Tröscher, A. R. et al. Microglial nodules provide the environment for pathogenic T cells in human encephalitis. Acta Neuropathol. 137, 619-635 (2019).

37. Safaiyan, S. et al. White matter aging drives microglial diversity. Neuron 109, 1100-1117 (2021).

38. Yuan, J., Amin, P. \& Ofengeim, D. Necroptosis and RIPK1-mediated neuroinflammation in CNS diseases. Nat. Rev. Neurosci. 20, 19-33 (2019).

39. Matute-Blanch, C. et al. Chitinase 3 -like 1 is neurotoxic in primary cultured neurons. Sci. Rep. 10, 7118 (2020).

40. Tremblay, R., Lee, S. \& Rudy, B. GABAergic interneurons in the neocortex: from cellular properties to circuits. Neuron 91, 260-292 (2016).

41. Petersen, C. C. H. \& Crochet, S. Synaptic computation and sensory processing in neocortical layer 2/3. Neuron 78, 28-48 (2013).

42. Al-Dalahmah, O. et al. Single-nucleus RNA-seq identifies Huntington disease astrocyte states. Acta Neuropathol. Commun. 8, 19 (2020).

43. Buniello, A. et al. The NHGRI-EBI GWAS catalog of published genome-wide association studies, targeted arrays and summary statistics 2019. Nucleic Acids Res. 47, D1005-D1012 (2019).

44. Hosseini, S. et al. Long-term neuroinflammation induced by influenza a virus infection and the impact on hippocampal neuron morphology and function. J. Neurosci. 38 3060-3080 (2018).

45. Deleidi, M. \& Isacson, O. Viral and inflammatory triggers of neurodegenerative diseases. Sci. Transl. Med. 4, 121ps3 (2012).

46. Zhou, Y. et al. Human and mouse single-nucleus transcriptomics reveal TREM2-dependent and TREM2-independent cellular responses in Alzheimer's disease. Nat. Med. 26, 131-142 (2020).

Publisher's note Springer Nature remains neutral with regard to jurisdictional claims in published maps and institutional affiliations.

(c) The Author(s), under exclusive licence to Springer Nature Limited 2021, corrected publication 2021 


\section{Methods}

No statistical methods were used to predetermine sample size. The experiments were not randomized, and investigators were not blinded to allocation during experiments and outcome assessment.

\section{Isolation of nuclei from frozen post-mortem medial frontal gyrus}

Frozen medial frontal cortex tissue from post-mortem control individuals and patients with COVID-19 was obtained from the Stanford/VA/NIA Ageing Clinical Research Center (ACRC) and the Saarland University Hospital Institute for Neuropathology, with approval from local ethics committees. Group characteristics are presented in Supplementary Table1. The protocol for the isolation of nuclei was adapted from previous studies ${ }^{5,25,46-48}$, and performed in a BSL2+ biosafety cabinet wearing personal protective equipment (PPE). All procedures were carried out on ice or at $4{ }^{\circ} \mathrm{C}$. In brief, $50 \mathrm{mg}$ of post-mortem brain tissue was dounce-homogenized in $2 \mathrm{ml}$ of Nuclei EZ Prep Lysis Buffer (Sigma,

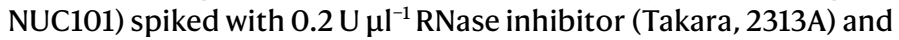
EDTA-free protease inhibitor Cocktail (Roche, 11873580001) before incubating on ice for $5 \mathrm{~min}$ in a final volume of $5 \mathrm{ml}$. Homogenized tissue was filtered through a 100- $\mu$ m cell strainer (Falcon, 352360), mixed with an equal volume of $50 \%$ iodixanol density gradient medium in PBS (OptiPrep, Sigma-Aldrich, D1556) to make a final concentration of $25 \%$ iodixanol. Thirty per cent iodixanol was layered underneath the $25 \%$ mixture. Similarly, $40 \%$ iodixanol was layered underneath the $30 \%$ iodixanol. In a swinging-bucket centrifuge, nuclei were centrifuged for $20 \mathrm{~min}$ at 3,000 r.c.f. After centrifugation, the nuclei were present at the interface of the $30 \%$ and $40 \%$ iodixanol solutions. Isolated nuclei were

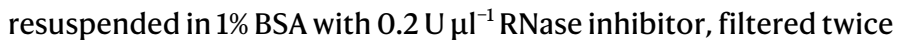
through a 40- $\mu \mathrm{m}$ strainer (Flowmi) and counted on an TC20 automated cell counter (Bio-Rad) after the addition of Trypan blue. We did not use statistical methods to predetermine sample sizes, but our sample sizes are similar to those reported in previous publications ${ }^{24,25,49}$.

\section{Isolation of nuclei from frozen post-mortem choroid plexus}

Frozen choroid plexus tissue was extracted from the lateral ventricles of post-mortem tissue obtained from the Stanford University Pathology department and the Saarland University Hospital Institute for Neuropathology, with approval from local ethics committees. Group characteristics are presented in Supplementary Table 1 . All procedures were carried out on ice or at $4{ }^{\circ} \mathrm{C}$, and in a BSL2+ biosafety cabinet while wearing PPE. Dounce homogenization or enzymatic dissociation resulted in loss of nuclei integrity and low nuclei complexity $(<50$ median genes per nuclei). We hypothesized that, similar to shaking an apple tree, gentle pipetting of choroid plexi tissue in lysis buffer could liberate nuclei without needing to physically disintegrate the fibrous choroid matrix-and thus avoid collateral physical damage to nuclei. Specifically, $40 \mathrm{mg}$ of choroid plexus tissue was thawed in $250 \mu \mathrm{l}$ of $1 \% \mathrm{BSA}$ with $0.2 \mathrm{U} \mathrm{\mu l}^{-1} \mathrm{RNase}$ inhibitor until the tissue settled. Five $\mathrm{ml}$ of lysis buffer $\left(10 \mathrm{mM}\right.$ Tris, $10 \mathrm{mM} \mathrm{NaCl}, 3 \mathrm{mM} \mathrm{MgCl}_{2}, 0.1 \%$ Nonidet

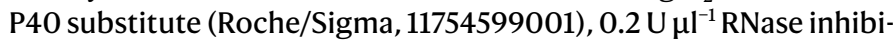
tor, and protease inhibitor) was added and tissue incubated on ice for $10 \mathrm{~min}$ with gentle swirling every $2 \mathrm{~min}$. Five $\mathrm{ml}$ of $1 \%$ BSA was added and the tissue triturated 10 times with a $5-\mathrm{ml}$ serological pipette. After centrifugation $(500 \mathrm{~g}, 5 \mathrm{~min})$, pelleted nuclei were resuspended in $1 \%$

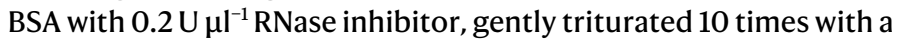
$1-\mathrm{ml}$ regular-bore pipette tip and filtered twice through a $70-\mu \mathrm{m}$ and then a 40- $\mu \mathrm{m}$ strainer (Flowmi). Debris was inspected on a brightfield microscope and nuclei were counted on an TC20 automated cell counter (Bio-Rad) after the addition of Trypan blue.

\section{Droplet-based snRNA-seq}

For droplet-based snRNA-seq, libraries were prepared using the Chromium Next GEM Single Cell 3'v.3.1 according to the manufacturer's protocol (10x Genomics), targeting 10,000 nuclei per sample after counting with a TC20 Automated Cell Counter (Bio-Rad). Thirteen cycles were applied to brain parenchyma samples to generate cDNA, and 15 for choroid plexus samples. All samples underwent 15 or 16 cycles for final library generation. Generated snRNA-seq libraries were sequenced across two S4 lanes on a NovaSeq 6000 (150 cycles, Novogene).

\section{snRNA-seq quality control}

Raw gene counts were obtained by aligning reads to the hg 38 genome (refdata-gex-GRCh38-2020-A) using CellRanger software (v.4.0.0) (10x Genomics). To account for unspliced nuclear transcripts, reads mapping to pre-mRNA were also counted. As previously published, a cut-off value of 200 unique molecular identifiers was used to select nuclei of sufficient complexity for further analysis ${ }^{5}$. As initial reference, the entire dataset was projected onto two-dimensional space using UMAP on the top 20 principal components ${ }^{50}$. Three approaches were combined for quality control: (1) ambient cell free mRNA contamination was removed using Soup $\mathrm{X}^{51}$ for each individual sample; (2) outliers with a high ratio of mitochondrial $(>5 \%,<200$ features) relative to endogenous RNAs and homotypic doublets ( $>5,000$ features) were removed in Seurat 3.2.152 and (3) after scTransform normalization and integration, doublets and multiplets were filtered out using DoubletFinder with subsequent manual inspection and filtering on the basis of cell-type-specific marker genes ${ }^{53}$. Similarly, genes detected in fewer than four cells were excluded from the analysis. The core statistical parameters of DoubletFinder ( $\mathrm{nExp}$ and $\mathrm{pK}$ ) used to build artificial doublets for true doublet classification were determined automatically using recommended settings. The computed $\mathrm{nExp}$ and $\mathrm{pK}$ values for each sample are provided in Supplementary Table 1. After applying these filtering steps, the dataset contained 65,309 high-quality nuclei.

\section{Cell annotations}

The SCTransform-based integration workflow of Seurat ${ }^{54}$ was used to align data, using default settings. In brief, the integration workflow searches for common gene modules (anchors) in cells with similar transcriptomes. Individual samples after undergoing quality control (described in 'snRNA-seq quality control') are integrated in a step-wise fashion, using cellular sequencing depth as a covariate to mitigate technical artefacts. After combining the samples into a single dataset or Seurat object, genes were projected into principal component space using the principal component analysis (RunPCA). The first 80 (for global object), 30 (choroid plexus) or 25 (specific cell types) dimensions were used as inputs into the FindNeighbours, FindClusters (at 0.2 resolution) and RunUMAP functions of Seurat. In brief, a shared-nearest-neighbour graph was constructed on the basis of the Euclidean distance metric in principal component space, and cells were clustered using the Louvain method. RunUMAP functions with default settings was used to calculate 2D UMAP coordinates and search for distinct cell populations. The positive differential expression of each cluster against all other clusters (MAST) was used to identify marker genes for each cluster ${ }^{55}$. We annotated cell types using previously published marker genes ${ }^{5,8,24,46}$. To distinguish between confounding (perivascular) macrophages and $T$ cells and pure microglia in the larger cortex immune population, we subset the cluster and repeated the standard steps of Seurat for dimension reduction and unsupervised clustering. Then, to yield pure microglia by requiring the normalized expression of the specific cell-type markers MRC1 and CD247 to be strictly less than 1. As choosing a threshold involves a sensitivity-specificity trade-off, we sought to set strict cut-offs as to yield pure microglia at a high specificity. The $\mathrm{MRC1}^{+}$cell population did not form separate clusters in an unsupervised clustering of the larger immune population and was not associated with better or lower library quality scores, as assessed through the number of detected unique molecular identifiers, number of detected RNAs (genes) and percentage of mitochondrial reads. 


\section{Differential gene expression and subcluster analysis}

Differential gene expression of genes comparing control individuals and patients with COVID-19-or comparing cell-type subcluster markers-was done using the MAST ${ }^{55}$ algorithm (v.1.12.0), which implements a two-part hurdle model, and has demonstrated superior type-I error control without significantly sacrificing sensitivity ${ }^{56-59}$. First, we ensured that our data did not exhibit signs of confounding effects (Extended Data Fig. 2). For example, although sex imbalance of patient cohorts can influence some genes in single-cell analysis and is a general issue in the field, we balanced genders by group, mitigating variance due to sex (Extended Data Fig. 2a, Supplementary Tables 3, 5). Default Seurat thresholds of log-transformed fold change $>0.25$ (absolute value), adjusted $P$ value (Bonferroni correction) $<0.05$ and expression in greater than $10 \%$ of cells were required to consider a gene differentially expressed, as similarly done in previous studies of the brain ${ }^{5,8,25,26,46,60-63}$ and COVID-19 $9^{64-70}$. Sex and batch were set as latent variables. Our sensitivity to detect DEGs for a given cell type was not driven by the number of nuclei isolated (Extended Data Fig. 4c).

Cell-quality-associated markers were removed and biological pathway and gene ontology enrichment analysis were performed using Enrichr ${ }^{71}$, Metascape ${ }^{72}$ or GeneTrail $3^{73}$ with input species set to Homo sapiens and using standard parameters. Docking, processing and viral defence genes relevant to SARS-CoV-2 were chosen on the basis of a previous publication ${ }^{16}$. To identify microglia subcluster markers, differential expression analysis of cells grouped in each subcluster was performed against the remaining cells within the given cell-type. Markers were defined based on the MAST algorithm using only positive values with log-transformed fold change $>0.25$ (absolute value) and adjusted $P$ value (Bonferroni correction) $<0.05$. Enrichment or over-representation of the overlap between markers defining the COVID-19 microglia 2 cluster and the Mathys ${ }^{5}$ Alzheimer's disease Mic1 cluster followed the hypergeometric probability, using the set of 17,926 protein-coding genes as background. To assess alternative differential expression approaches, raw gene counts were aggregated for each sample and cell-type cluster separately. For the subsequent pseudobulk analysis, we used the pbDS function of the muscat package $^{74}$ with limma-voom ${ }^{75}$ selected as differential state method, and the parameters min_cells, and filter set to 20 and gene, respectively, where we configured sample sex and batch as latent variables in the design matrix. All other parameters were kept as default.

\section{RT- $q$ PCR validation of snRNA-seq differential gene expression}

For RT-qPCR validation of our snRNA-seq DEG analysis, we focused on choroid plexus tissue because of its relative homogeneity compared to cortex: epithelium and mesenchymal cells form over $90 \%$ of all nuclei and, hence, DEGs in those cell types can be assessed even in bulk choroid plexus mRNA samples with only an approximately $10 \%$ potential confound from other cell types. This is not the case with cortex samples consisting of various cell types and subtypes (for example, neuronal subtypes). In brief, choroid plexus nuclei were isolated as in 'Isolation of nuclei from frozen post-mortem choroid plexus', and bulk mRNA isolated using the RNeasy Micro Kit (Qiagen). cDNA was generated using the qScript cDNA SuperMix (Quantabio) and then mixed with SYBR green master mix before loading as technical duplicates on a LightCycler 480 (Roche). $\Delta \Delta C_{\mathrm{T}}$ values normalized to $A C T B$ were used to assess relative gene expression between samples. The following validated primer pairs were used (PrimerBank, human $)^{76}$ to assess major inflammatory genes predicted upregulated in COVID-19 as well as other genes predicted upregulated at a similar log-transformed fold change to confirm the validity of default snRNA-seq DEG MAST thresholds:IFITM3:5'-CTGGGCTTC ATAGCATTCGCCT-3' (forward) and 5'-AGATGTTCAGGCACTTGGCGGT-3' (reverse); STAT3: 5'-CAGCA GCTTGACACACGGTA-3' (forward) and 5'-AAACACCAAAGTGG CATGTGA-3' (reverse); C7: 5'-AATGGCTGTACCAAGACTCAGA-3' (forward) and 5'-GCTGATGCACTGACCTGAAAA-3' (reverse); NQO1: 5'-GAAGAGCACTGATCGTACTGGC-3' (forward) and 5'-GGATACTGAA AGTTCGCAGGG-3' (reverse);ZFP36:5'-GACTGAGCTATGTCGGACCTT-3' (forward) and 5'-GAGTTC CGTCTTGTATTTGGGG-3' (reverse); SDC4: 5'-GGACCTCCTAGAAGGCCGATA-3' (forward) and 5'-AGGGC CGATCATGGAGTCTT-3' (reverse); ACTB: 5'-CACCATTGGCAA TGAGCGGTTC-3' (forward) and 5'-AGGTCTTTGCGGATGTCCACGT-3' (reverse); and (housekeeping performed in duplicate): 5'-GGAGAAGAGCTACGAGCTGCCTGAC-3' (forward) and 5'-AAGGTAGTTTCGTGGATGCCACAGG-3' (reverse)

\section{Monocle trajectory analysis}

Monocle3 (v.0.2.1.) was used to generate the pseudotime trajectory analysis in microglia ${ }^{77}$. Cells were reclustered as described in 'Cell annotations' and used as input into Monocle to infer cluster and lineage relationships within a given cell type. Specifically, UMAP embeddings and cell subclusters generated from Seurat were converted to a cell_data_set object using SeuratWrappers (v.0.2.0) and then used as input to perform trajectory graph learning and pseudotime measurement through reversed graph embedding with Monocle.

\section{Viral transcript analysis}

Four RNA-based approaches were applied to systematically probe for the presence of SARS-CoV-2 RNA in the brain: analysis by snRNA-seq, bulk RNA-seq after viral isolation (QIAamp Viral RNA Mini Kit, Qiagen, manufacturer's instructions), bulk RNA-seq after whole transcriptome isolation and RT-PCR using US Centers of Disease Control and Prevention (CDC) Emergency Use Authorization primers against the SARS-CoV-2 N1 and N2 genes (IDT 10006770). Both bulk RNA-seq RNA underwent established cDNA and library generation: in brief, mRNA was transcribed into full-length cDNA by using the Smart-Seq v.4 Ultra-Low-Input RNA kit from Clontech according to the manufacturer's instructions. Samples were validated with an Agilent 2100 Bioanalyzer. Full-length cDNA was processed with the Nextera XT kit from Illumina for library preparation according to the manufacturer's protocol. Library quality was verified with an Agilent 2100 Bioanalyzer. Sequencing was carried out on a NovaSeq 6000 (150 cycles, Novogene). For RT-PCR analysis, bulk choroid whole transcriptome mRNA samples were diluted and mixed with SYBR green master mix before loading as technical duplicates on a LightCycler 480 (Roche) for 40 cycles.

To search for SARS-CoV-2 reads in either the snRNA-seq or bulk RNA-seq datasets, raw .fastq files were subjected to read alignment via Viral-Track ${ }^{78}$, VIRTUS ${ }^{79}$ or centrifuge ${ }^{80}$ using the human (GRCh38) genome reference. For Viral-Track, both a collection of 12,163 consensus virus sequences from Virusite ${ }^{81}$ (release 2020.3) and 17,133 curated SARS-CoV-2 genomes from NCBI (downloaded on 29 September 2020) were used. For centrifuge, a preprocessed virus index compiled by genexa containing among other viruses 138 SARS-CoV-2 genomes was used. We also adopted a complementary approach ${ }^{82}$ focusing on SARS-CoV-2 reads, in which barcoded but unmapped BAM reads were aligned using STAR to the SARS-CoV-2 reference genome, with a less stringent mapping parameter (outFilterMatchNmin 25-30) than the original Viral-Track pipeline.

\section{Cell-cell communication}

Cell-cell interactions based on the expression of known ligand-receptor pairs in different cell types were inferred using CellChat ${ }^{32}$ (v.0.02). To identify potential cell-cell communication networks perturbed or induced in brains of patients with COVID-19, we followed the official workflow and loaded the normalized counts into CellChat and applied the preprocessing functions identifyOverExpressedGenes, identifyOverExpressedInteractions and projectData with standard parameters set. As database, we selected the Secreted Signalling pathways and used the precompiled human Protein-protein-Interactions as a priori network information. For the main analyses the core functions 
computeCommunProb, computeCommunProbPathway and aggregateNet were applied using standard parameters and fixed randomization seeds. Finally, to determine the senders and receivers in the network, the function netAnalysis_signallingRole was applied on the netP data slot.

\section{Overlap with GWAS hits}

From the GWAS catalogue ${ }^{43}$, we obtained GWAS risk genes for neurological disorders (Alzheimer's disease, amyotrophic lateral sclerosis, brain ageing, multiple system atrophy, multiple sclerosis, Parkinson's disease and narcolepsy), psychiatric disorders (attention deficit hyperactivity disorder, autism, bipolar disorder, depression, psychosis, post-traumatic stress disorder and schizophrenia) and neurobehaviour traits (anxiety, suicidality, insomnia, neuroticism, risk behaviour, intelligence and cognitive function). We removed gene duplicates and GWAS loci either not reported or in intergenic regions, and used a $P<9 \times 10^{-6}$ to identify significant associations ${ }^{25}$. Then, as GWAS signals can point to multiple candidate genes within the same locus, we focused on the 'Reported Gene(s)' (genes reported as associated by the authors of each GWAS study). Disorders and traits exhibiting a significant number of genes that were also perturbed in patients with COVID-19 are highlighted. Following gene symbol extraction, we curated the gene set by (1) removing unknown or outdated gene names using the HGNChelper package (v.0.8.6), (2) converting remaining Ensembl gene identifiers to actual gene names using the packages ensembldb (v.2.10.0) and EnsDb. Hsapiens.v86 (v.2.99.0) and (3) removing any remaining duplicates. We then calculated the overlap between each set of GWAS genes with the cell-type-specific DEGs. Finally, a statistical enrichment of each overlap against background was calculated using a hypergeometric test with the total background size set equal to the number of unique RNAs mapped in our dataset $(29,431)$. Overlaps between GWAS DEGs and disease GWAS genes expressed were calculated separately for each cell type.

\section{Comparison of DEGs in chronic CNS disease}

We compiled cell type-specific DEGs reported in published datasets for Alzheimer's disease ${ }^{5}$, autism spectrum disorder ${ }^{8}$, Huntington's disease ${ }^{42}$ and multiple sclerosis ${ }^{26}$. Lists of gene symbols were curated using the aforementioned approach. COVID-19 DEGs that overlap with those found across the selected CNS diseases were called shared, whereas those not previously reported were called unique to COVID-19. Statistical significance calculations of over-representation in DEG overlaps are based on cumulative hypergeometric $P$ values analogous to the procedures described in 'Differential gene expression and sub-cluster analysis' and 'Overlap with GWAS hits', with the total background size set equal to the number of unique RNAs mapped in our dataset $(29,431)$. Using the smaller set of 17,926 protein-coding genes as background does not change the qualitative statistical significance of the overlaps. Similar to the analysis of GWAS hits, we determined the overlap and tested its significance for each cell type separately.

\section{Principal variance component and principal component analyses}

In brief, to conduct the principal variance component analysis (PVCA), we aggregated the SoupX corrected raw counts for each gene and each biological sample using the aggregateData function of the muscat package (v.1.2.1) ${ }^{74}$. The resulting matrix was normalized by dividing each feature of a sample by the total counts from that sample, multiplied by 100,000 and scaling the result using the function $\log (x+1)$. As variables we considered the sample annotation fields 'Sample-ID', 'Patient-ID', 'Sex', 'Brain-region', 'Disease', 'ageBin', 'nNucleiBin' and 'Batch'. As PVCA is designed to support factors, we assigned the values for numeric variables into ordered bins, more specifically, into six half-open (left-closed) intervals of size 1,000 starting at 1,000 for the number of nuclei and five similarly defined intervals of size 10 starting at 51 for the age. We set the cut-off for the minimal variance out of the total variance being explained to be $95 \%$. For each single annotation variable, or first higher-order combinations of such, a cut-off of 0.005 was applied to consider them explanatory. All variables (or combinations of such) not passing the threshold were summarized as Other in the analysis. The residual was then defined as the remaining proportion of variance not being associated with any of the variables that are explanatory nor informative to a minor proportion. To conduct principal component analysis, we aggregated the log-normalized cell counts from Seurat for each gene and sample using the aggregateData function from muscat and centred the gene expression vectors before computing eigenvectors.

\section{Computational analysis, statistics and schematics}

Analysis of the data was performed with the statistical programming language R (v.3.6.3) using the following general-purpose package for loading, saving and manipulating data, as well as generating plots, and fitting statistical models: dplyr (v.1.0.0), ggplot2 (v.3.2.2.), patchwork (v.1.0.1), openxlsx (v.4.1.5), bioconductor-scater (v.1.14.6) ${ }^{83}$, bioconductor-dropletutils $(\mathrm{v} 1.6 .1)^{84,85}$, bioconductor-complexheatmap (v.2.2.0 $)^{86}$, tidyverse (v.1.3.0) $)^{87}$ and lsa (v.0.73.2). All other tasks were performed on an x86_64-based Ubuntu (4.15.0-55-generic kernel) server cluster. We did not use statistical methods to predetermine sample sizes, but they are similar to those reported in previous publications ${ }^{24,25,49}$. Data in graphs are always presented as mean \pm s.e.m. Statistical tests used for group or cluster comparisons in bulk or single-nucleus RNA-seq experiment analysis are specified in the respective sections in Methods. Schematic diagrams were created with BioRender.com.

\section{Immunohistochemistry}

Paraffin-embedded human brain tissue (medial frontal cortex, meninges and choroid plexus) adjacent to tissue processed for snRNA-seq was subjected to immunohistochemistry.

After deparaffinization and rehydration of $1-3-\mu \mathrm{m}$ sections, peroxidases were blocked by incubation in $1 \% \mathrm{H}_{2} \mathrm{O}_{2}$ for $15 \mathrm{~min}$ at room temperature. Heat antigen retrieval was performed by steaming at $98^{\circ} \mathrm{C}$ in target retrieval solution pH 6.1 (Dako, no. S1699) for $30 \mathrm{~min}$. Sections were allowed to cool down at room temperature. Following antigen retrieval, sections were incubated for $45 \mathrm{~min}$ at room temperature with the anti-SARS spike glycoprotein antibody $3 \mathrm{~A} 2$ (rabbit, Abcam ab272420, 1:100), which has been used in previous publications $^{17,18}$, anti-SARS-CoV-2 spike antibody (mouse, GeneTex GTX632604, 1 A9 clone, 1:100) used in a previous publication ${ }^{19}$, anti-SARS-CoV-2 spike antibody (rabbit, Sino Biological 40150-T62-CoV2, 1:100), anti-SARS-CoV-2 nucleoprotein antibody (rabbit, Sino Biological 40143-T62, 1:100) used in a previous publication ${ }^{30}$, and anti-human CD68 (mouse, Dako M0876, PG-M1 clone, 1:100) for determining microglial reactivity. Both antibodies were diluted in Dako REAL antibody diluent no. S2022. After three washes with wash buffer (Dako no. S3006), the Dako REAL EnVision HRP kit (no. K5007) or alkaline phosphatase/ RED kit (no. K500511) was used for the visualization of the antibody reaction according to the manufacturer's instructions. Sections were counterstained with Mayer's haemalum (Sigma-Aldrich no. 1.09249). After dehydration, coverslips were mounted with Entellan (Merck no. 1.07961). Images were acquired with an Olympus BX 40 microscope, equipped with an Olympus SC30 digital microscope camera using the Olympus cellSens software. To assess disease-associated innate immune activation in brains of individuals with COVID-19, slides were screened at low magnification and areas with the most pronounced changes were used for quantification. Spatial context was used to determine the myeloid cell type-for example, the meninges are evident in a brain slice, enabling confident identification of resident $\mathrm{CD} 68^{+}$cells as meningeal, and likewise for the brain vasculature. A semiquantitative categorization for activation, as typical in pathology, was used: mild = detectable microgliosis, atypical for healthy tissue; moderate $=\mathrm{a}$ 
pathological process typical of pathological changes; severe = a marked pathological process. Several clusters of microglia or macrophages were characterized as excessive beyond the severe category.

\section{Reporting summary}

Further information on research design is available in the Nature Research Reporting Summary linked to this paper.

\section{Data availability}

Raw sequencing data are deposited under NCBI Gene Expression Omnibus (GEO) GSE159812. Normalized count data are also available for download at https://twc-stanford.shinyapps.io/scRNA_Brain_COVID19. Any other relevant data are available from the corresponding authors upon reasonable request. Source data are provided with this paper.

\section{Code availability}

All analyses have been carried out using freely available software packages. Custom code used to analyse the RNA-seq data and datasets generated and/or processed in the current study is available from the corresponding authors upon request.

47. Swiech, L. et al. In vivo interrogation of gene function in the mammalian brain using CRISPR-Cas9. Nat. Biotechnol. 33, 102-106 (2015).

48. Corces, M. R. et al. An improved ATAC-seq protocol reduces background and enables interrogation of frozen tissues. Nat. Methods 14, 959-962 (2017).

49. Zhong, S. et al. A single-cell RNA-seq survey of the developmental landscape of the human prefrontal cortex. Nature 555, 524-528 (2018)

50. McInnes, L., Healy, J., Saul, N. \& Großberger, L. UMAP: uniform manifold approximation and projection. J. Open Source Softw. 3, 861 (2018).

51. Young, M. D. \& Behjati, S. SoupX removes ambient RNA contamination from droplet-based single-cell RNA sequencing data. Gigascience $\mathbf{9}$, giaa151 (2020).

52. Satija, R., Farrell, J. A., Gennert, D., Schier, A. F. \& Regev, A. Spatial reconstruction of single-cell gene expression data. Nat. Biotechnol. 33, 495-502 (2015).

53. McGinnis, C. S., Murrow, L. M. \& Gartner, Z. J. DoubletFinder: doublet detection in single-cell RNA sequencing data using artificial nearest neighbors. Cell Syst. 8, 329-337 (2019).

54. Hafemeister, C. \& Satija, R. Normalization and variance stabilization of single-cell RNA-seq data using regularized negative binomial regression. Genome Biol. 20, 296 (2019).

55. Finak, G. et al. MAST: a flexible statistical framework for assessing transcriptional changes and characterizing heterogeneity in single-cell RNA sequencing data. Genome Biol. 16, 278 (2015).

56. Soneson, C. \& Robinson, M. D. Bias, robustness and scalability in single-cell differential expression analysis. Nat. Methods 15, 255-261 (2018).

57. Wang, T., Li, B., Nelson, C. E. \& Nabavi, S. Comparative analysis of differential gene expression analysis tools for single-cell RNA sequencing data. BMC Bioinformatics 20, 40 (2019).

58. Mou, T., Deng, W., Gu, F., Pawitan, Y. \& Vu, T. N. Reproducibility of methods to detect differentially expressed genes from single-cell RNA sequencing. Front. Genet. 10, 133 (2020).

59. Dal Molin, A., Baruzzo, G. \& Di Camillo, B. Single-cell RNA-sequencing: assessment of differential expression analysis methods. Front. Genet. 8, 62 (2017).

60. Ximerakis, M. et al. Single-cell transcriptomic profiling of the aging mouse brain. Nat. Neurosci. 22, 1696-1708 (2019).

61. The Tabula Muris Consortium. A single-cell transcriptomic atlas characterizes ageing tissues in the mouse. Nature 583, 590-595 (2020).

62. Yang, A. C. et al. Physiological blood-brain transport is impaired with age by a shift in transcytosis. Nature 583, 425-430 (2020).

63. Chen, M. B. et al. Brain endothelial cells are exquisite sensors of age-related circulatory cues. Cell Rep. 30, 4418-4432 (2020).

64. Wilk, A. J. et al. A single-cell atlas of the peripheral immune response in patients with severe COVID-19. Nat. Med. 26, 1070-1076 (2020).

65. $\mathrm{Xu}, \mathrm{G}$. et al. The differential immune responses to COVID-19 in peripheral and lung revealed by single-cell RNA sequencing. Cell Discov. 6, 73 (2020).

66. Schulte-Schrepping, J. et al. Severe COVID-19 is marked by a dysregulated myeloid cell compartment. Cell 182, 1419-1440 (2020).

67. Guo, C. et al. Single-cell analysis of two severe COVID-19 patients reveals a monocyte-associated and tocilizumab-responding cytokine storm. Nat. Commun. 11, 3924 (2020).
68. Meckiff, B. J. et al. Imbalance of regulatory and cytotoxic SARS-CoV-2-reactive CD4 T cells in COVID-19. Cell 183, 1340-1353 (2020).

69. Lee, J. S. et al. Immunophenotyping of COVID-19 and influenza highlights the role of type I interferons in development of severe COVID-19. Sci. Immunol. 5, eabd1554 (2020).

70. Su, Y. et al. Multi-omics resolves a sharp disease-state shift between mild and moderate COVID-19. Cell 183, 1479-1495 (2020).

71. Chen, E. Y. et al. Enrichr: interactive and collaborative HTML5 gene list enrichment analysis tool. BMC Bioinformatics 14, 128 (2013).

72. Zhou, Y. et al. Metascape provides a biologist-oriented resource for the analysis of systems-level datasets. Nat. Commun. 10, 1523 (2019).

73. Gerstner, N. et al. GeneTrail 3: advanced high-throughput enrichment analysis. Nucleic Acids Res. 48, W515-W520 (2020).

74. Crowell, H. L. et al. muscat detects subpopulation-specific state transitions from multi-sample multi-condition single-cell transcriptomics data. Nat. Commun. 11, 6077 (2020).

75. Law, C. W., Chen, Y., Shi, W. \& Smyth, G. K. voom: precision weights unlock linear model analysis tools for RNA-seq read counts. Genome Biol. 15, R29 (2014).

76. Spandidos, A., Wang, X., Wang, H. \& Seed, B. PrimerBank: a resource of human and mouse PCR primer pairs for gene expression detection and quantification. Nucleic Acids Res. 38, D792-D799 (2010).

77. Trapnell, C. et al. The dynamics and regulators of cell fate decisions are revealed by pseudotemporal ordering of single cells. Nat. Biotechnol. 32, 381-386 (2014).

78. Bost, P. et al. Host-viral infection maps reveal signatures of severe COVID-19 patients. Cell 181, 1475-1488 (2020).

79. Yasumizu, Y., Hara, A., Sakaguchi, S. \& Ohkura, N. VIRTUS: a pipeline for comprehensive virus analysis from conventional RNA-seq data. Bioinformatics btaa859 (2020).

80. Kim, D., Song, L., Breitwieser, F. P. \& Salzberg, S. L. Centrifuge: rapid and sensitive classification of metagenomic sequences. Genome Res. 26, 1721-1729 (2016).

81. Stano, M., Beke, G. \& Klucar, L. viruSITE-integrated database for viral genomics. Database 2016, baw162 (2016)

82. Wauters, E. et al. Discriminating mild from critical COVID-19 by innate and adaptive immune single-cell profiling of bronchoalveolar lavages. Cell Res. 31, 272-290 (2021).

83. McCarthy, D. J., Campbell, K. R., Lun, A. T. L. \& Wills, Q. F. Scater: pre-processing, quality control, normalization and visualization of single-cell RNA-seq data in R. Bioinformatics 33, 1179-1186 (2017)

84. Griffiths, J. A., Richard, A. C., Bach, K., Lun, A. T. L. \& Marioni, J. C. Detection and removal of barcode swapping in single-cell RNA-seq data. Nat. Commun. 9, 2667 (2018).

85. Lun, A. T. L. et al. EmptyDrops: distinguishing cells from empty droplets in droplet-based single-cell RNA sequencing data. Genome Biol. 20, 63 (2019).

86. Gu, Z., Eils, R. \& Schlesner, M. Complex heatmaps reveal patterns and correlations in multidimensional genomic data. Bioinformatics 32, 2847-2849 (2016).

87. Wickham, H. et al. Welcome to the Tidyverse. J. Open Source Softw. 4, 1686 (2019). https://doi.org/10.21105/joss.01686.

88. Lan, X., Han, X., Li, Q., Yang, Q. W. \& Wang, J. Modulators of microglial activation and polarization after intracerebral haemorrhage. Nat. Rev. Neurol. 13, 420-433 (2017).

Acknowledgements We thank N. Khoury, T. Iram, E. Tapp and other members of the laboratories of T.W.-C. and A.K. for feedback and support, and H. Zhang and K. Dickey for laboratory management. This work was funded by the NOMIS Foundation (T.W.-C.), the National Institute on Aging (T32-AG0047126 to A.C.Y. and 1RF1AG059694 to T.W.-C.), Nan Fung Life Sciences (T.W.-C.), the Bertarelli Brain Rejuvenation Sequencing Cluster (an initiative of the Stanford Wu Tsai Neurosciences Institute) and the Stanford Alzheimer's Disease Research Center (P30 AG066515). A.C.Y. was supported by a Siebel Scholarship. F.K., G.P.S., T.F., W.J.S.-S. and A.K. are a part of the CORSAAR study supported by the State of Saarland, the Saarland University and the Rolf M. Schwiete Stiftung.

Author contributions A.C.Y., F.K., A.K. and T.W.-C. conceptualized the study. M.W.M., N. Ludwig, I.C., W.J.S.-S., N.S., D.C., D.B. and A.C.Y. provided and organized tissue samples. A.C.Y. performed tissue dissociations. A.C.Y., N.S., D.P.L., R.T.V., D.G., N. Lu, O.H. and K.C. prepared libraries for sequencing. A.C.Y. and M.R.A. performed RT-PCR. F.K., G.P.S., T.F. and A.C.Y. performed computational analysis, with F.K. leading advanced analysis and data management. P.M.L. developed the searchable web interface (Shiny app). J.A.S. and W.J.S.-S. performed immunohistochemical stains and antibody tests. A.C.Y., F.K. and C.A.M. assembled figures. A.C.Y. wrote the manuscript with input from all authors. F.K. and T.W.-C. edited the manuscript. T.W.-C. and A.K. supervised the study.

Competing interests T.W.-C. is a co-founder and scientific advisor of Alkahest Inc.

Additional information

Supplementary information The online version contains supplementary material available at https://doi.org/10.1038/s41586-021-03710-0.

Correspondence and requests for materials should be addressed to A.K. or T.W.-C. Peer review information Nature thanks the anonymous reviewers for their contribution to the peer review of this work.

Reprints and permissions information is available at http://www.nature.com/reprints. 


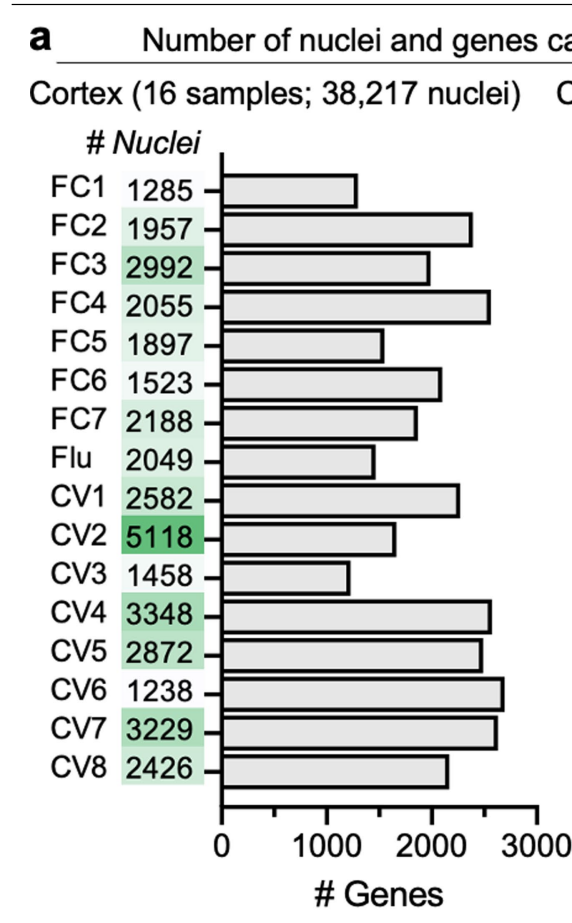

b Nuclei quality/ complexity by disease group Cortex
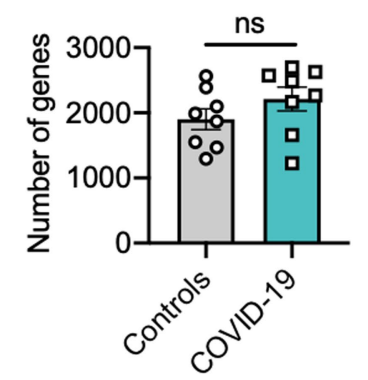

C

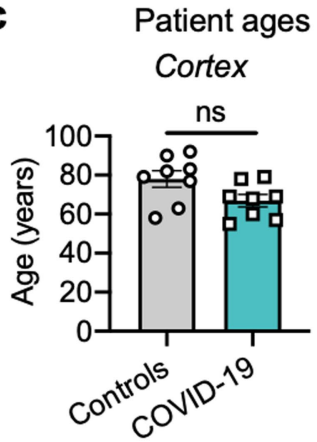

Choroid
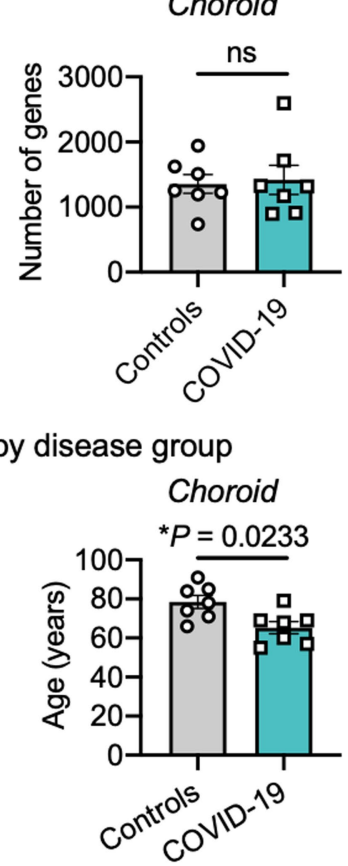

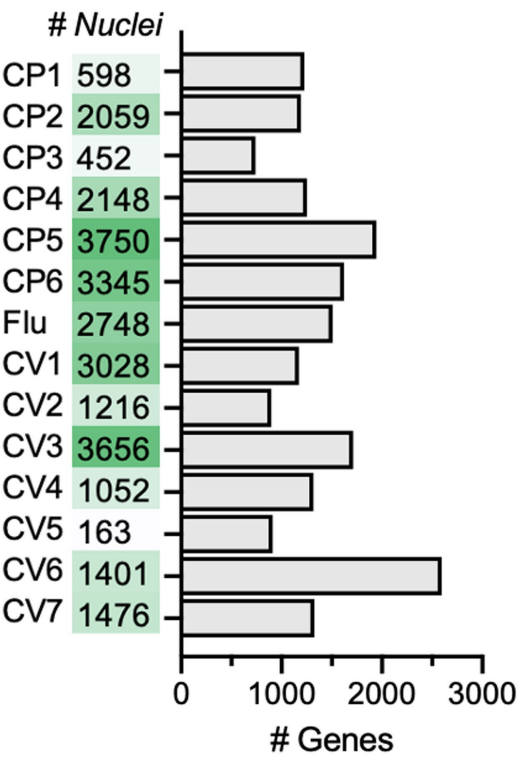

d Frontal cortical cell type proportions by disease

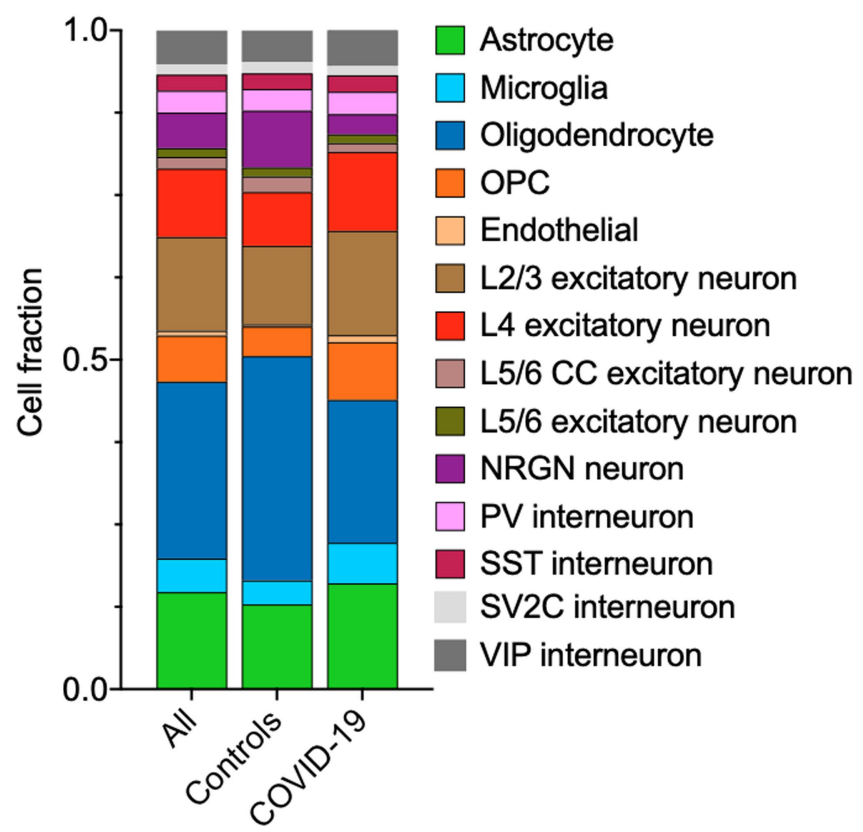

Extended Data Fig. 1 | Characterization of human cortical and choroid plexi nuclei sequenced. a, Total number of nuclei and median number of genes of each human sample sequenced in medial frontal cortex and choroid plexus. b, c, Quantification of the median number of genes detected per nuclei (b) and patient ages (c) in control (non-viral and influenza) and COVID-19 samples in e Choroid plexus cell type proportions by disease

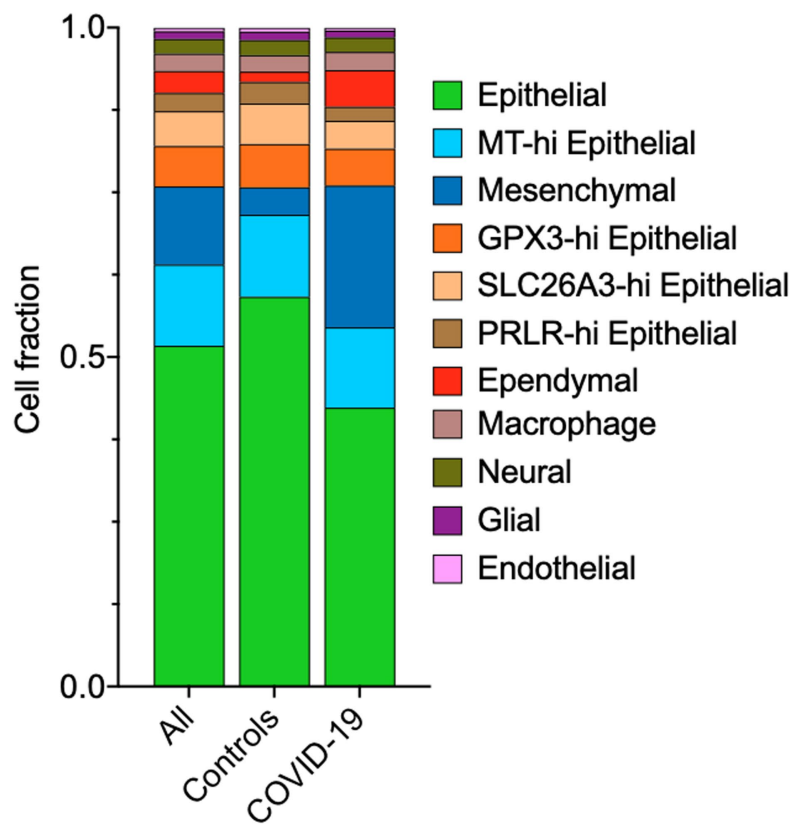

medial frontal cortex ( $n=8$ control; $n=8$ COVID-19, two-sided Mann-Whitey $t$-test; mean \pm s.e.m.) and choroid plexus ( $n=7$ control; $n=7$ COVID-19, two-sided Mann-Whitey $t$-test; mean \pm s.e.m.).d, e, Bar graph presenting frequency of nuclei for control and COVID-19 medial frontal cortex (d) and choroid plexus (e) sample groups. 
a

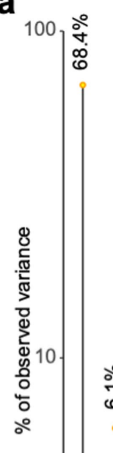

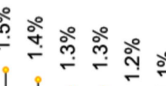

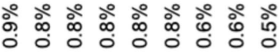

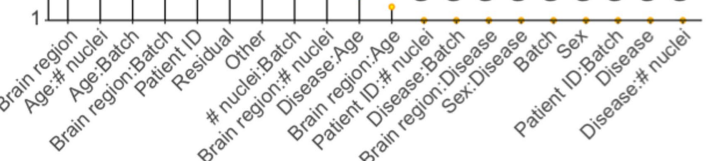

Cells of the medial frontal cortex

C

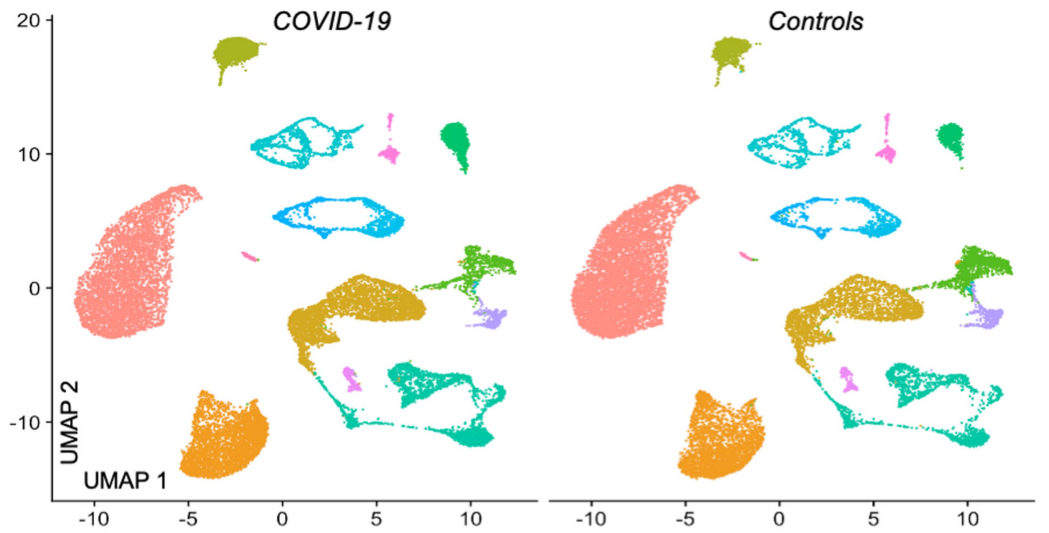

Cells of the choroid plexus
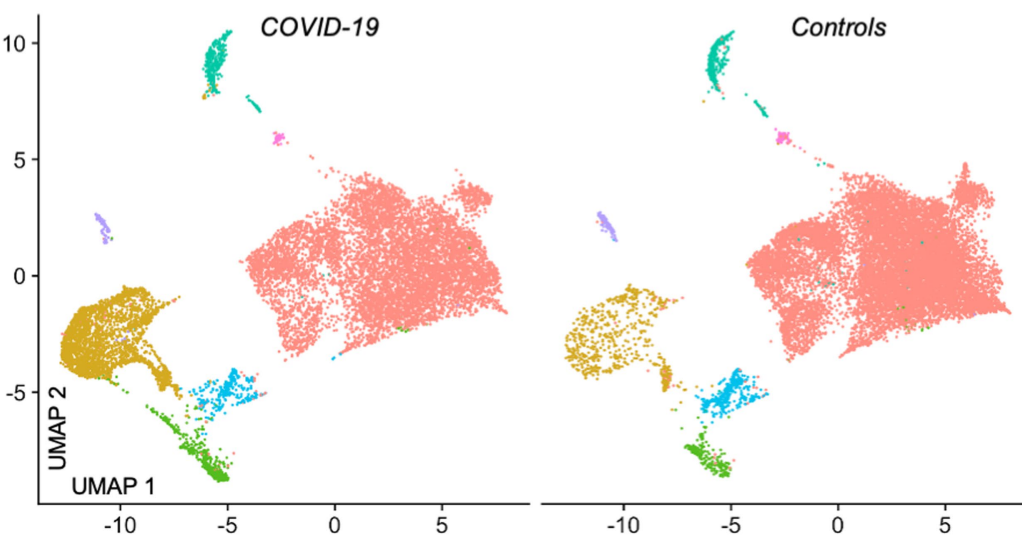

- Oligodendrocyte

- Astrocyte

L2/3 excitatory neuron

- OPC

- NRGN neuron

- Microglia

- L4 excitatory neuron

- VIP interneuron

- PV interneuron

- SST interneuron

- L5/6 CC excitatory neuron

- L5/6 excitatory neuron

- SV2C interneuron

- Endothelial
Extended Data Fig. 2 |Gene expression variance analysis. a, PVCA displaying the gene expression variance explained by residuals (biological and technical noise) or experimental factors such as brain region, age, sex and respective combinations. $n=30$ samples. $\mathbf{b}$, Principal component $(\mathrm{PC})$ analysis visualization of all samples, based on unscaled counts. c, UMAP projections of nuclei isolated from the medial frontal cortex (top) or choroid plexus (bottom), and split by disease group, showing no systematic batch effects. 
a Markers for parenchymal cells in the human frontal medial cortex

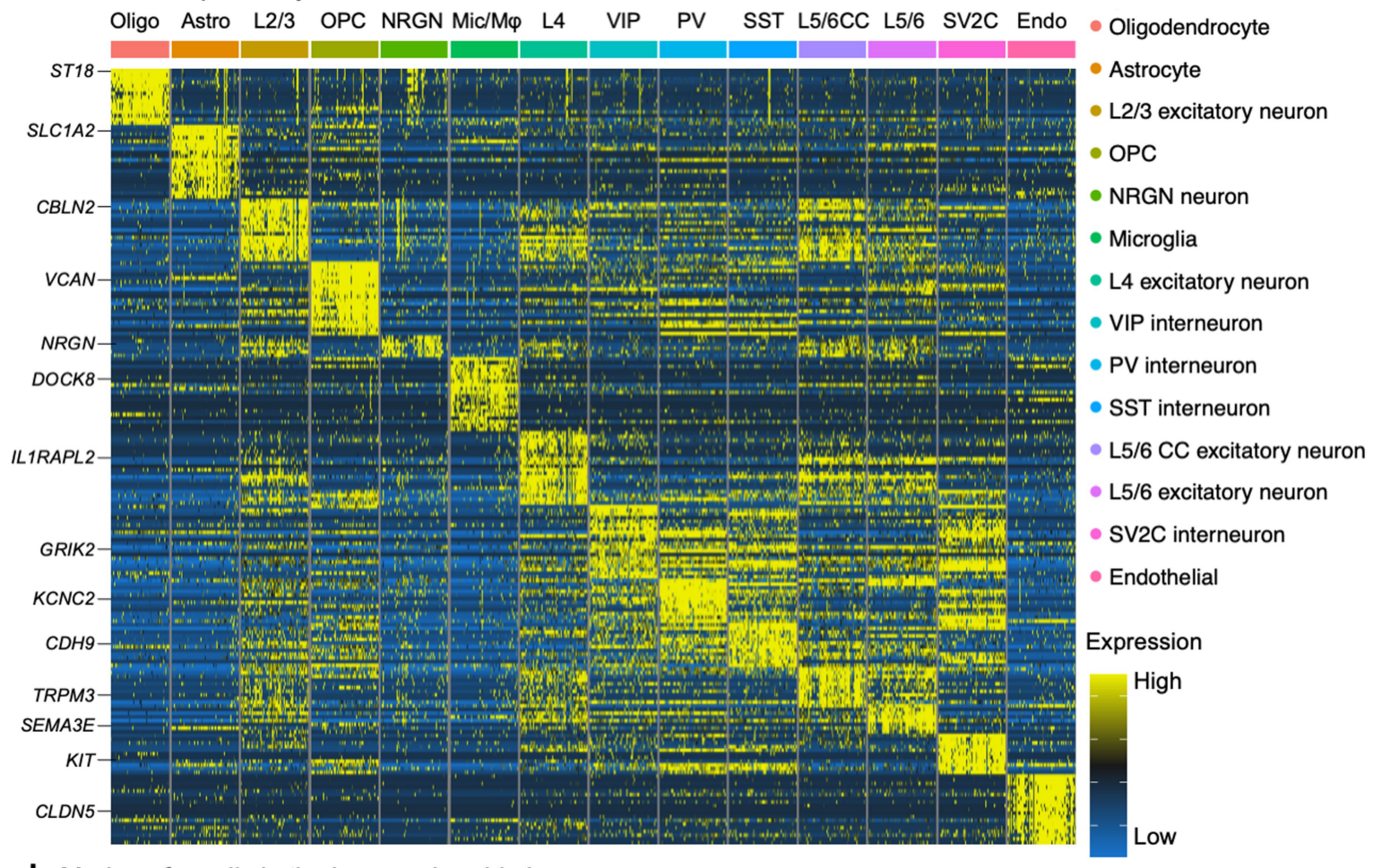

b Markers for cells in the human choroid plexus
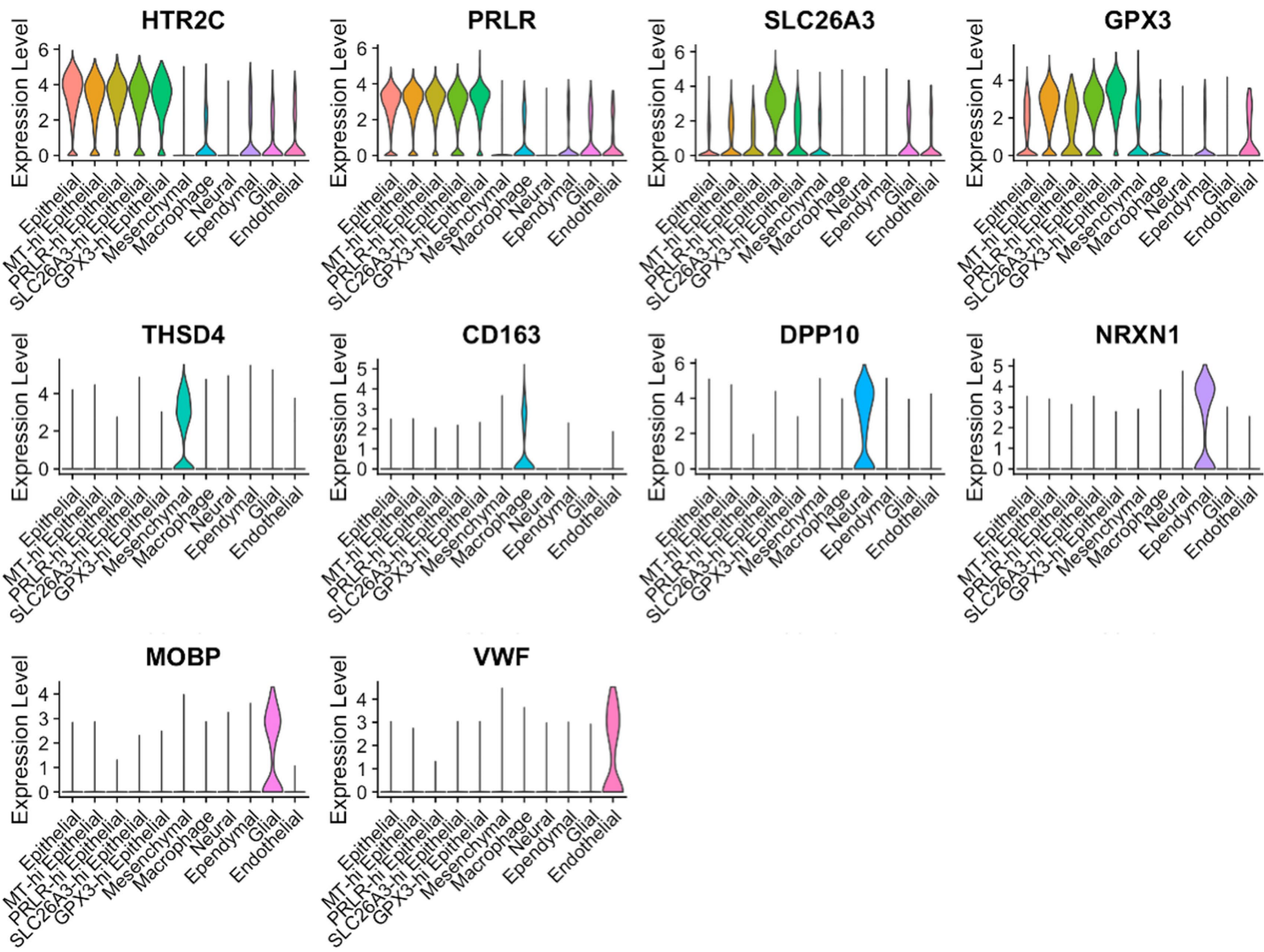

Extended Data Fig. $3 \mid$ Human brain cell-type markers. a, Top cell-typespecific genes across the types of cells captured in the human cortex. The colour bar indicates gene expression from low (blue) to high (yellow).

b, Example of top cell-type-specific genes across the types of cells captured in the human choroid plexus. Violin plots are centred around the median, with their shape representing cell distribution. 
a Significantly perturbed pathways across brain cell types in COVID-19

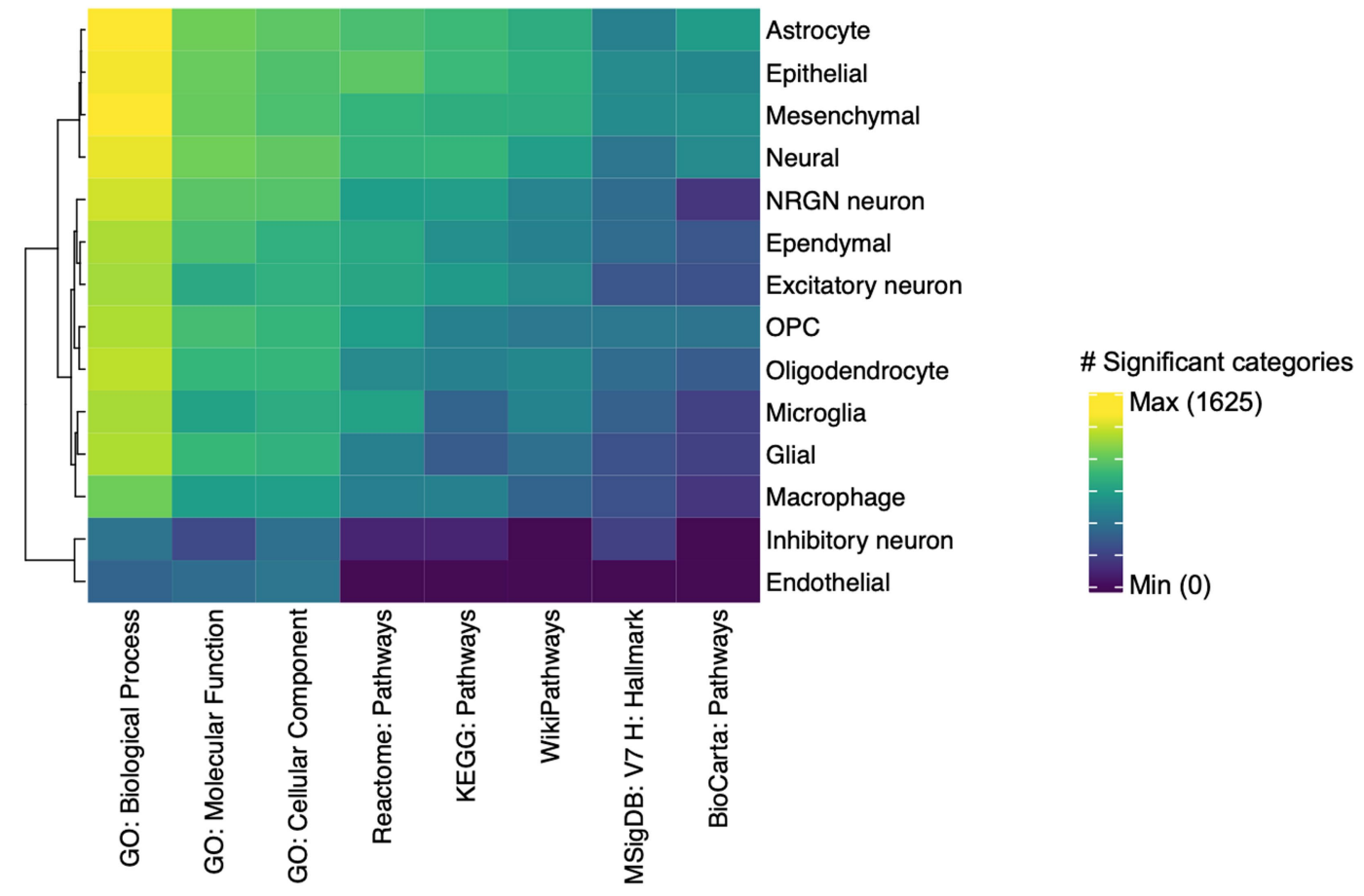

b Example perturbations in astrocytes with COVID-19

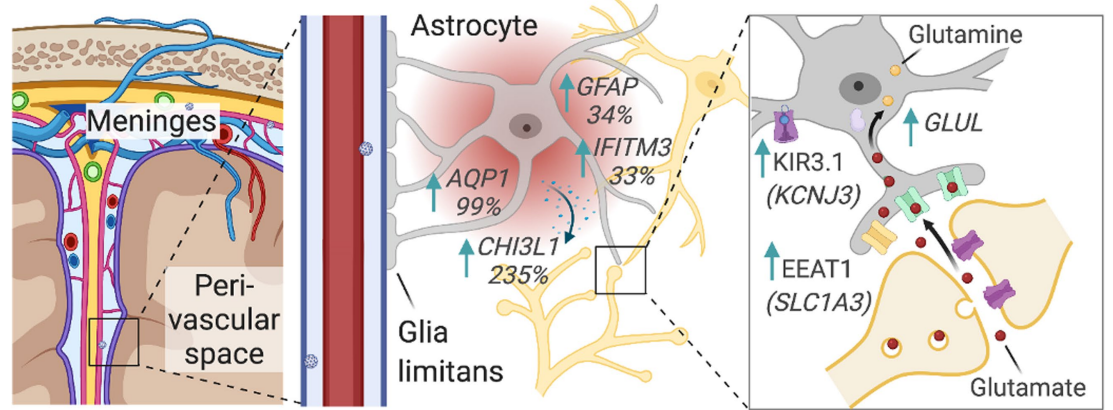

C Sensitivity for DEG detection is not driven by number of nuclei per cell type

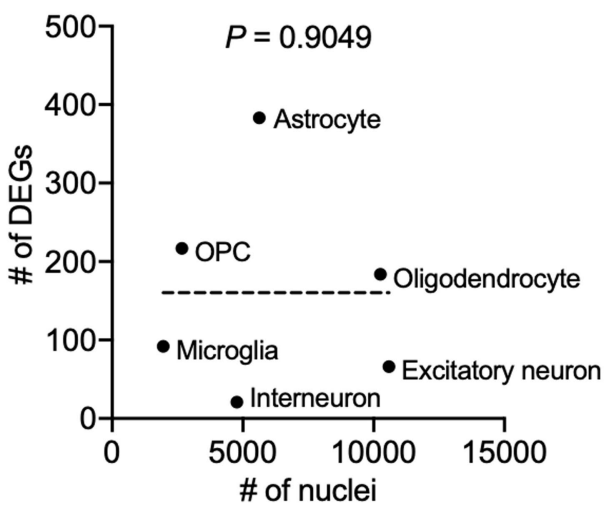

Extended Data Fig. 4 | Cell-type-specific changes in gene expression and intercellular signalling in the brain of individuals with CovID-19. a, Heat map displaying the number of significant biological pathways among the set of DEGs in each cell type (FDR $<0.05$, Benjamini-Hochberg adjustment, hypergeometric test). Number of significant pathways is indicated in graded black (low) to yellow (high). b, Example upregulation of inflammatory and dysregulation of homeostatic genes in COVID-19 astrocytes. c, Comparison of the number of nuclei isolated per cell type and the number of predicted DEGs. Two-sided $P$-value indicates the significance of the correlation (Pearson, not significant). 
a Brain parenchymal cells

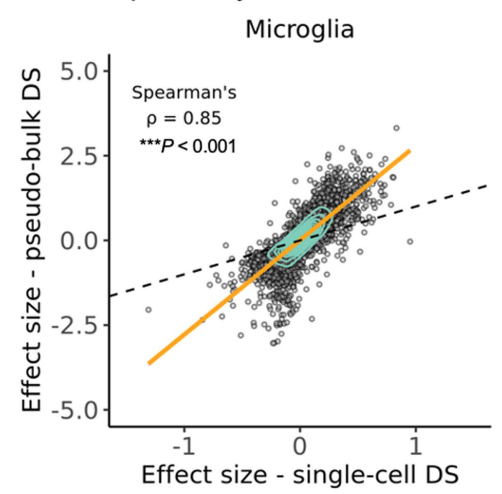

OPC

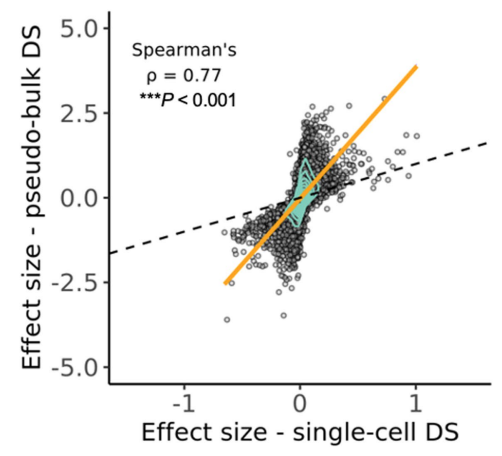

\section{b Choroid plexus cells}

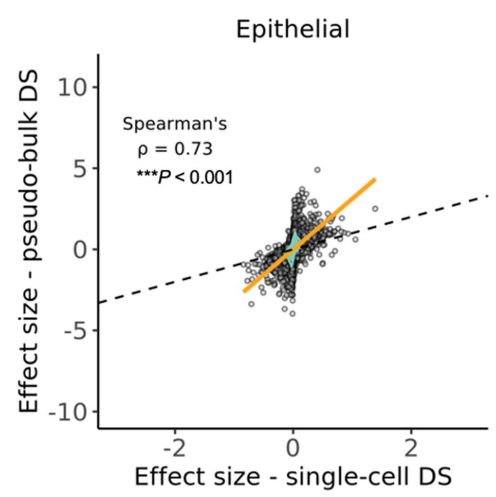

Macrophage

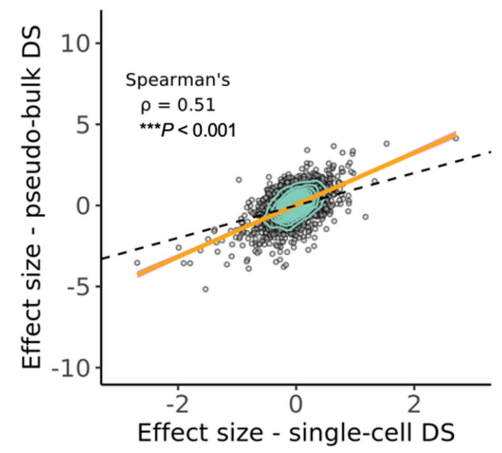

Extended Data Fig. 5 | Overlap between alternative snRNA-seq differential expression analysis methods. a, b, Scatter plots demonstrating the strong correlation between the calculated effect sizes of two differential gene expression analysis methods (MAST ${ }^{55}$ (used here) and pseudobulk ${ }^{74,75}$ ) across
Astrocyte

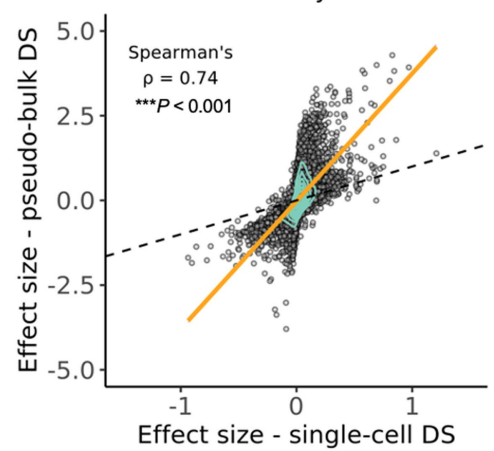

Excitatory neuron
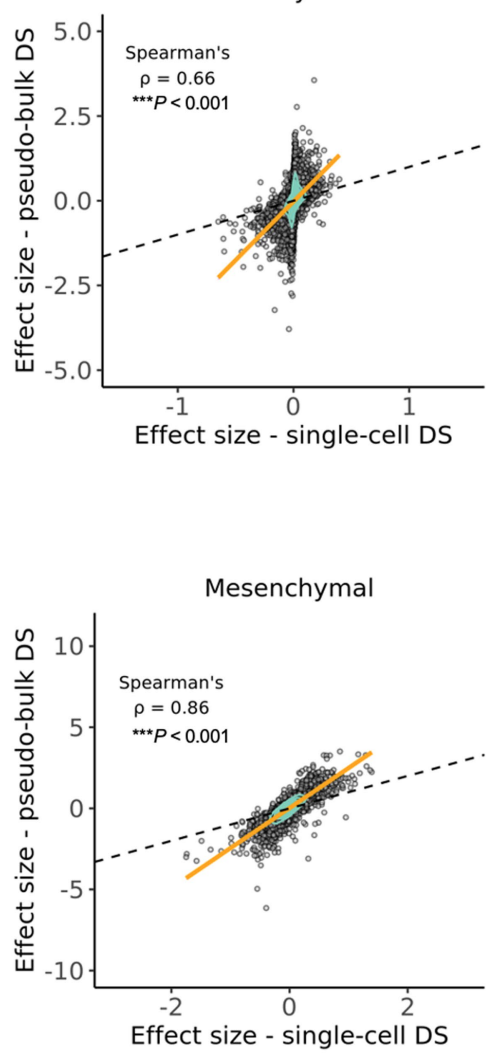

Neural

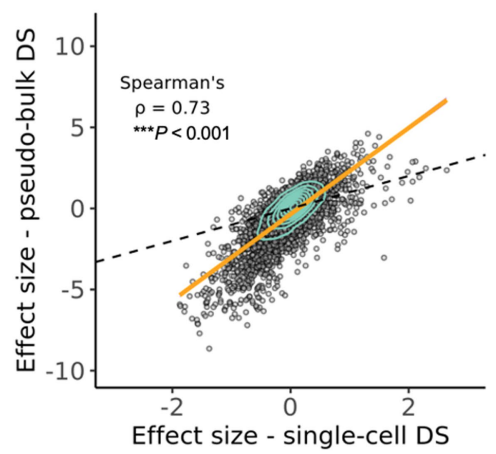

Oligodendrocyte

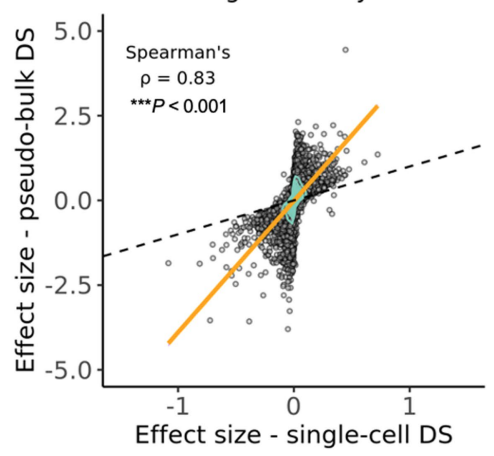

Inhibitory neuron

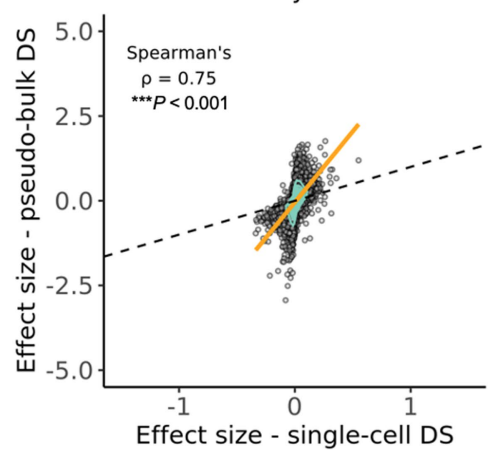

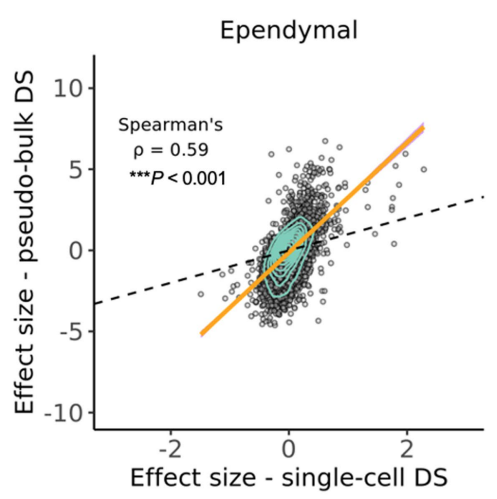

Glial

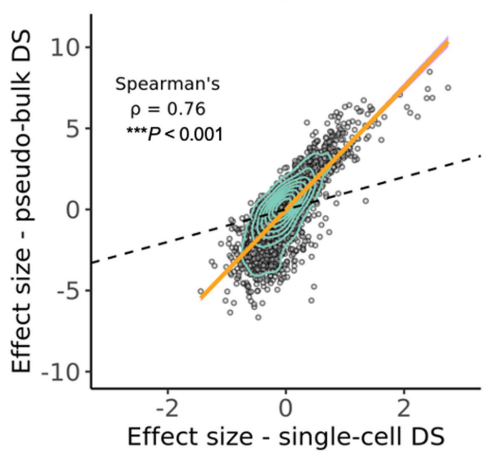

cell types in the human medial frontal cortex (a) and choroid plexus (b). Orange line denotes the trend line fitted with a generalized linear model, surrounded by a $95 \%$ confidence interval in purple. Spearman correlation is shown along with the significance by two-sided $P$-values. 
a Comparison of $\mathrm{PMI}-$ sensitive genes and COVID-19 DEGs, $P=\mathbf{P} \mathbf{0 . 9 5 0 1}$

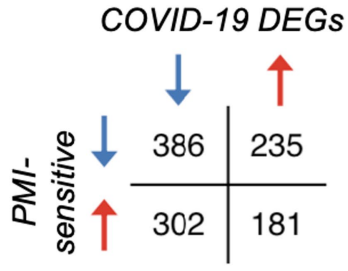

b Comparison by Dachet et al. categories
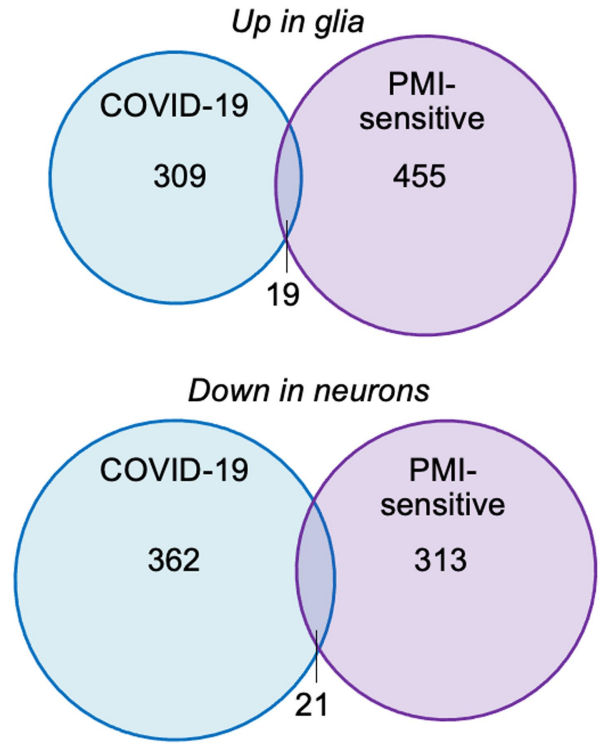

C PMI-sensitive genes do not vary significantly in analyzed COVID-19 brain PMI-

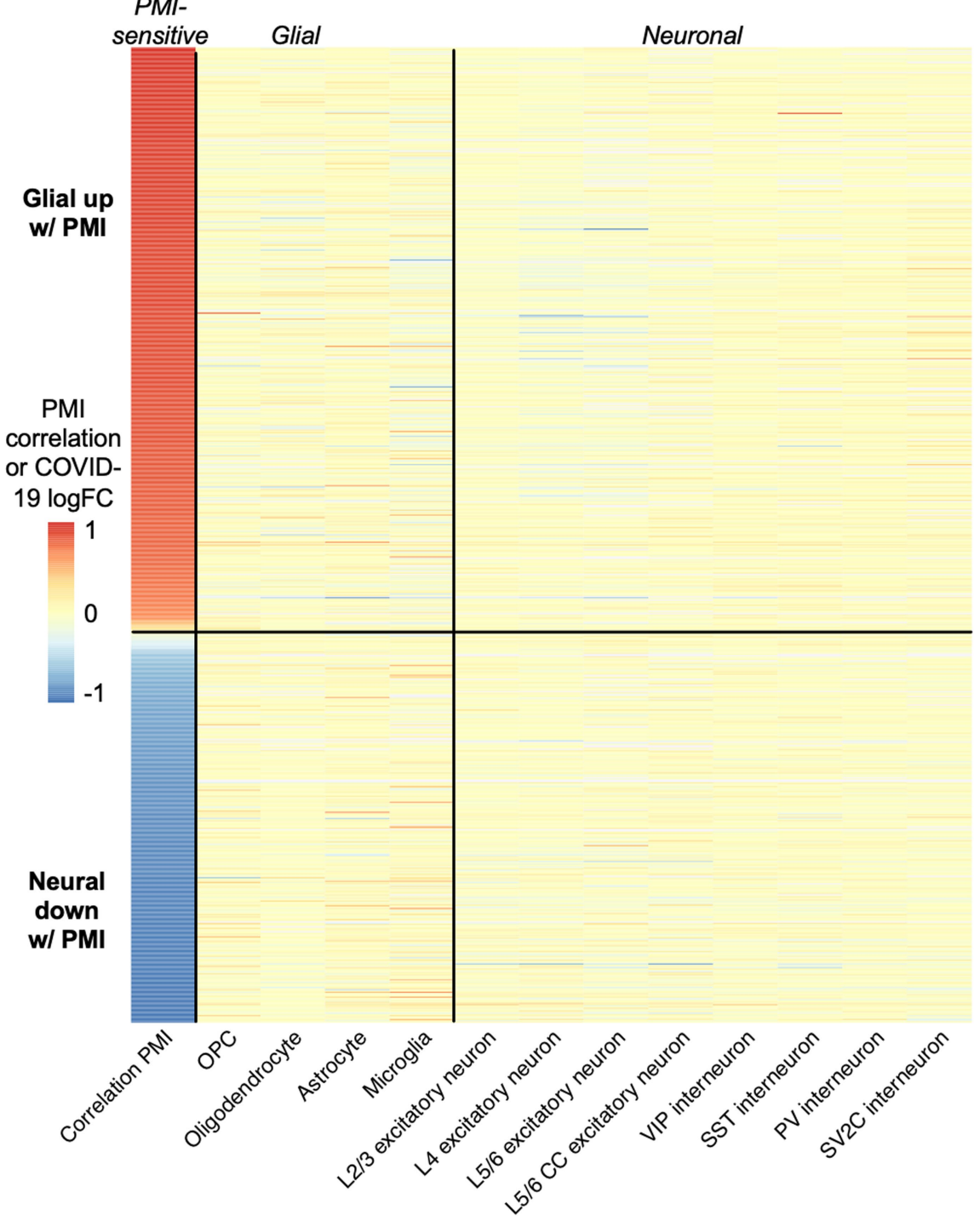

neural genes downregulated. Minimal overlap is seen with COVID-19 changes of the same category (for example, glial genes upregulated in COVID-19 versus glial genes upregulated with extended PMI).c. Heat map showing that PMI-sensitive genes are not the DEGs in COVID-19 and thus not driving the DEG-based findings of our study. significant overlap is observed (Fisher's exact test). b. The previous study ${ }^{27}$

categorized PMI-sensitive genes in two categories: glial genes upregulated and
Extended Data Fig. 6 | DEGs in the brains of individuals with COVID-19 show no significant overlap with brain PMI-sensitive genes. a, Comparison of post-mortem interval (PMI)-sensitive genes (left column, from a previous publication $^{27}$ ) and COVID-19 DEGs (all other columns). No statistically ( 
a Frontal medial cortex, parenchymal cells
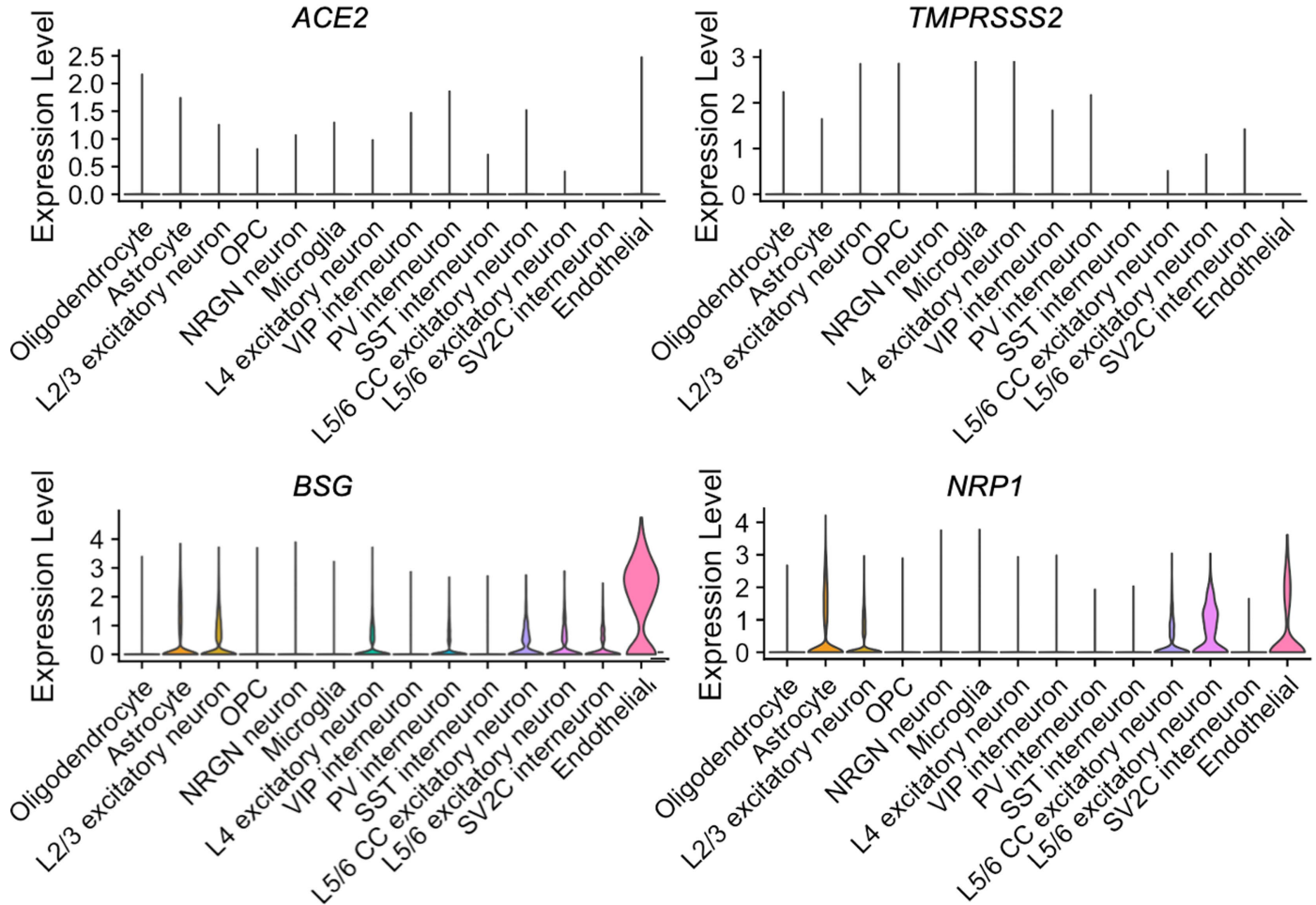

\section{b Choroid plexus cells}
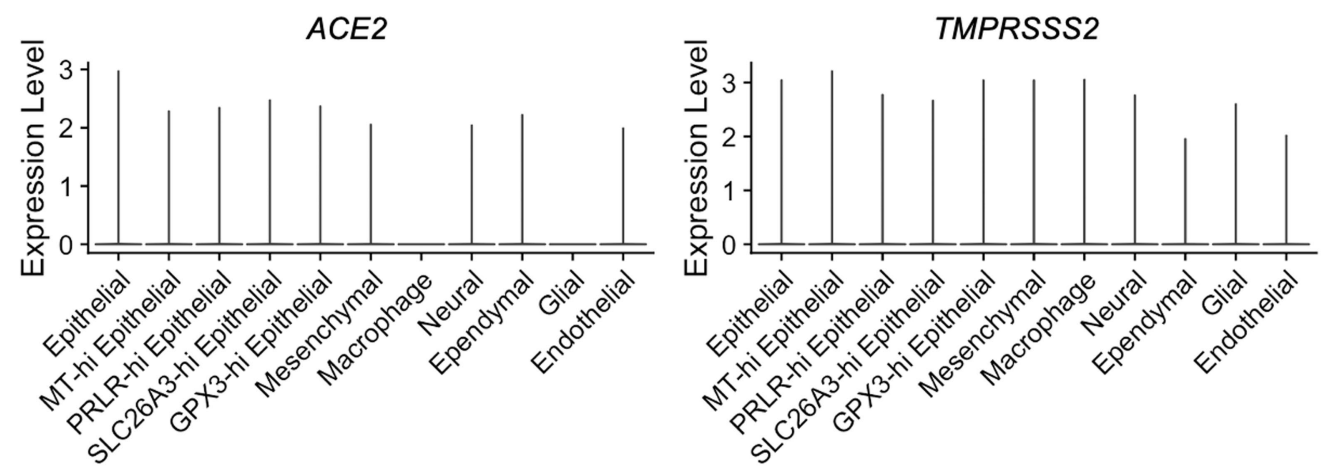

BSG
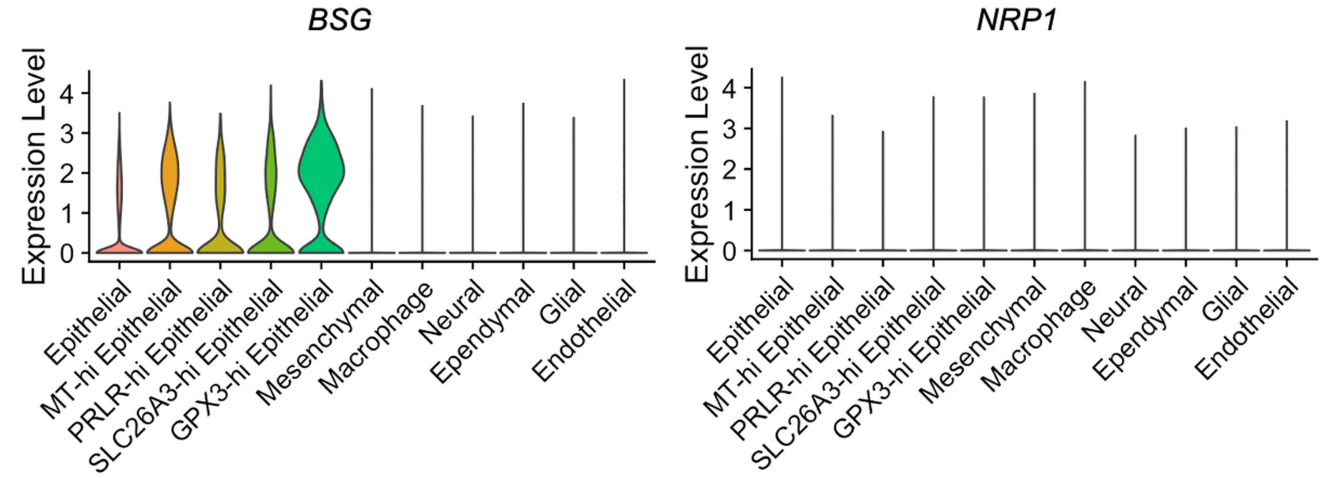

Extended Data Fig. 7 | Expression of SARS-CoV-2 virus entry genes across cell types. a, b, Expression of SARS-CoV-2 entry receptors, established and putative, across cell types in the human medial frontal cortex (a) and choroid

plexus (b). Violin plots are centred around the median, with their shape representing cell distribution. 
Macrophage activation in the COVID-19 choroid plexus

Haematoxylin CD68

Control patient

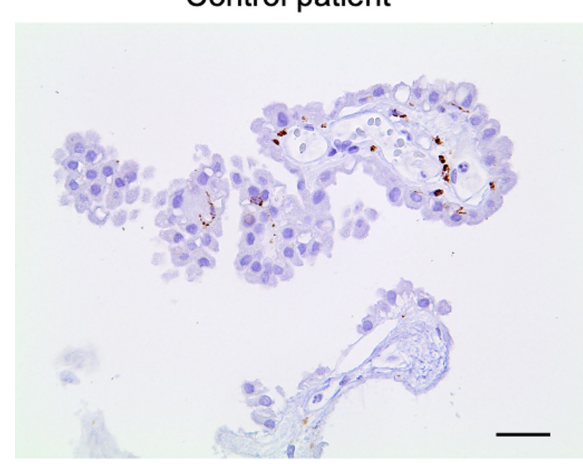

COVID-19 Patient 1

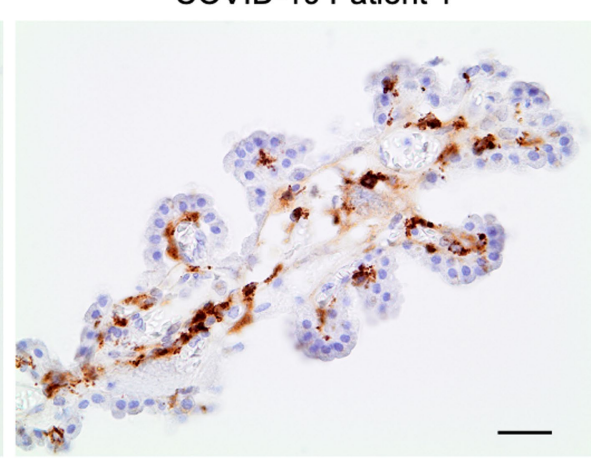

COVID-19 Patient 2

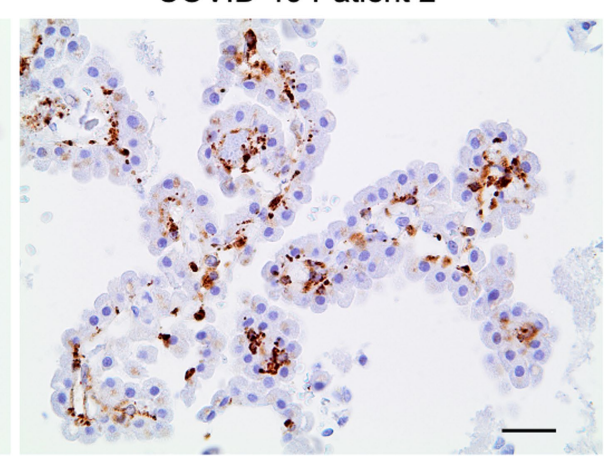

Extended Data Fig. $8 \mid$ Choroid plexus inflammation in COVID -19. Immunohistochemical staining for the macrophage activation marker CD 68 (brown) in the choroid plexus of patients with COVID-19 and control individuals. Haematoxylin counterstain (blue). Scale bars, $20 \mu \mathrm{m}$. 
a Summary, no specific signal above controls

\begin{tabular}{c|c|c} 
Detection method & $\begin{array}{c}\text { Choroid } \\
\text { plexus }\end{array}$ & Cortex \\
\hline $\begin{array}{c}\text { Single-nucleus } \\
\text { RNA-seq }\end{array}$ & $0 / 7 \mid 0 / 7$ & $0 / 8 \mid 0 / 8$ \\
\hline $\begin{array}{c}\text { Bulk RNA-seq } \\
\text { (Viral isolate) }\end{array}$ & $0 / 7 \mid 0 / 4$ & $0 / 5 \mid 0 / 4$ \\
\hline $\begin{array}{c}\text { Bulk RNA-seq } \\
\text { (Whole) }\end{array}$ & $0 / 6 \mid 0 / 7$ & \\
\hline RT-PCR & $0 / 6 \mid 0 / 7$ &
\end{tabular}

Controls COVID-19

\section{b Choroid plexus \\ RT-PCR, EUA primers}

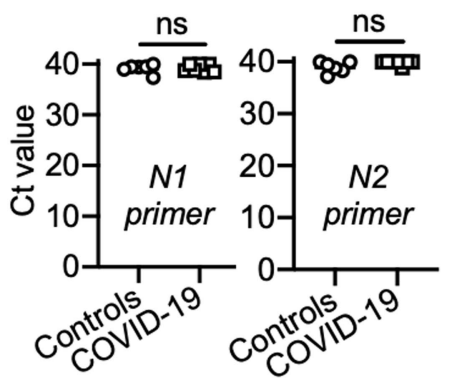

C $\quad a-S P I K E ~(3 A 2) A b$ reactivity in the human brain vasculature

d Choroid plexus \& meninges reactivity Haematoxylin Spike protein (HRP detection)
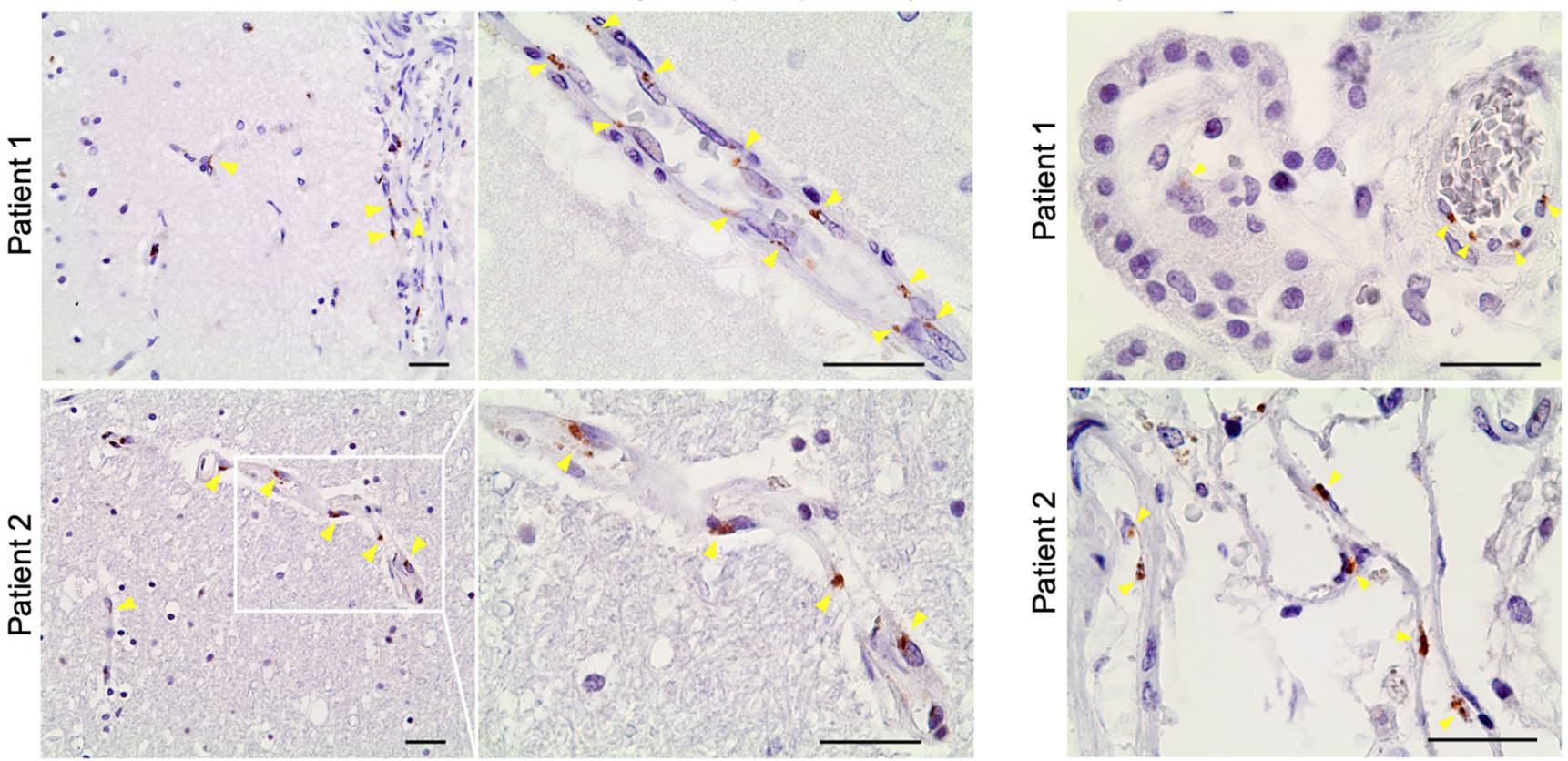

e Specific reactivity persists despite different secondary detection method

Haematoxylin Spike protein (biotin-AP detection)
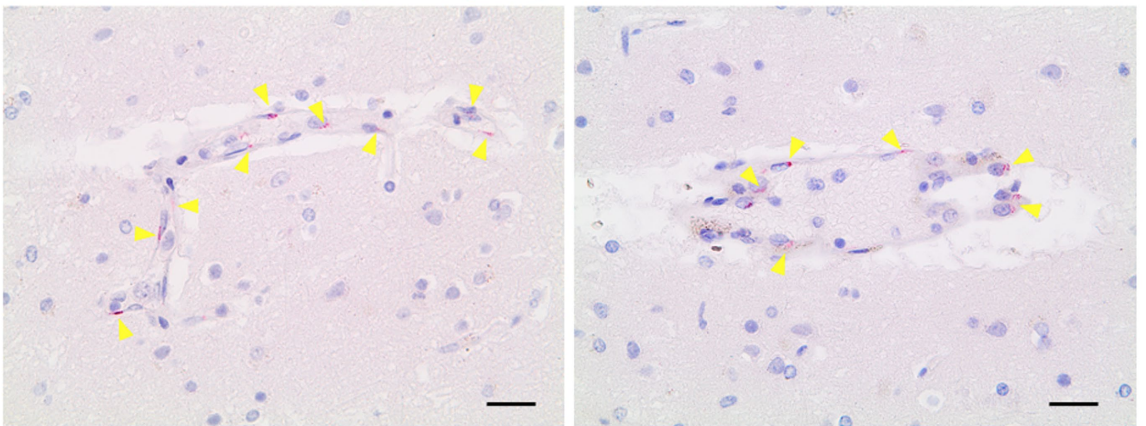

\section{Extended Data Fig. 9 | No conclusive detection of SARS-CoV-2}

neuroinvasion. a, Summary of RNA-based assays to detect SARS-CoV-2 in the human cortex and choroid plexus. Aside from the 3A2 antibody, no other anti-SARS-CoV-2 antibody detected viral protein antigen in the brain or choroid plexus. b, qPCR detection of the SARS-CoV-2 genes $N 1$ and $N 2$ via CDC Emergency Use Authorization primers on choroid plexus samples $(n=6$ non-viral control, $n=7$ COVID-19; two-sided Mann-Whitney $t$-test; mean \pm s.e.m.).c, Aberrant anti-SARS-CoV-2 spike (3A2) antibody reactivity (brown) in the frontal medial cortex of two patients with COVID-19 in tissue immediately adjacent to that used for snRNA-seq. Haematoxylin counterstain (purple). Scale bar, $20 \mu \mathrm{m}$.d, As in c, but for the choroid plexus and meninges in two patients with COVID-19. Scale bar, $20 \mu \mathrm{m}$.e, As in c, but using a different secondary antibody detection method (biotin-alkaline phosphatase (red)), recapitulating the specific vascular-localized signal. Scale bar, $20 \mu \mathrm{m}$. Immunohistochemical stains are representative of at least two independent experiments. 


\section{Choroid plexus inflammatory signaling into the brain}
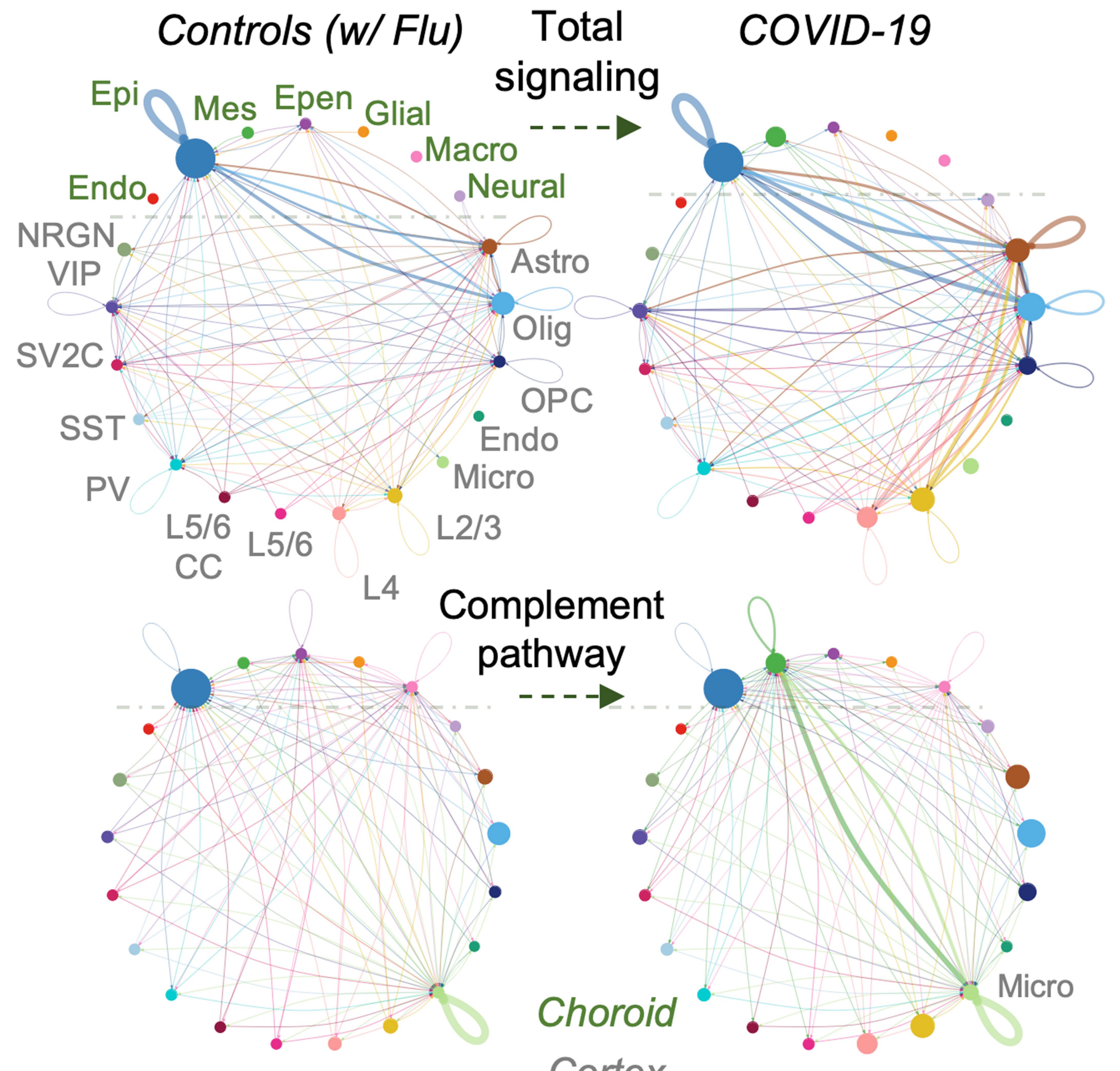

\section{Cortex}

Extended Data Fig. 10 | Cell communication analysis results for integrated choroid plexus and brain parenchyma cell types. Circle plot showing the number of statistically significant intercellular signalling interactions for total signalling (over 30 ligand-receptor pathways) and the complement family of molecules in control individuals (non-viral and influenza) compared to patients with COVID-19 (permutation test, CellChat ${ }^{34} ; n=8$ control, including influenza; $n=8$ COVID-19 for cortex; and $n=7$ control, including influenza; $n=7$
COVID-19 for choroid plexus). Each circle (colour) represents one cell type, and edges connecting circles represent significant intercellular signalling inferred between those cell types. Circles and edges were normalized and scaled to display relative sizes, with the former proportional to the number of cells from a given cell type and the latter according to the inferred strength of signalling. Cell type labels correspond to signalling pathway increased in COVID-19. 


\section{Article}

\section{Haematoxylin CD68}

\section{a Parenchymal microglial activation}
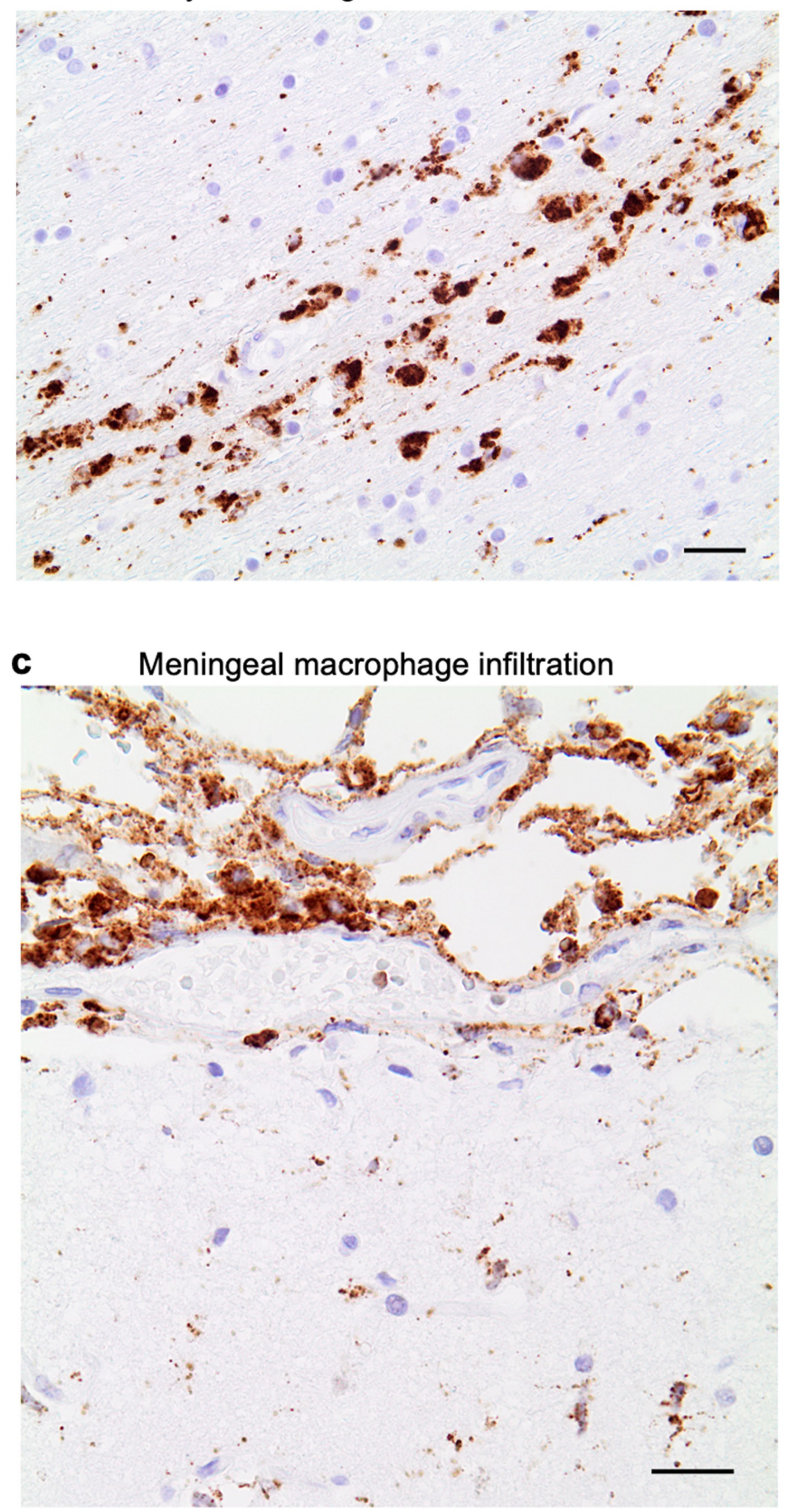

Extended Data Fig.11 | Activation of parenchymal microglia and perivascular macrophages in COVID-19. Immunohistochemical staining of microglia and macrophages by an antibody against the pro-inflammatory marker $\mathrm{CD} 68^{88}$ (immunoreaction in brown). Counterstained with haematoxylin for cell nuclei in blue. a, The frontal medial gyrus of patients with COVID-19 immediately adjacent to that used for snRNA-seq. A cluster of activated microglia up to single macrophages is immunostained in the parenchyma of the gyrus (subcortical white matter). Scale bar, $20 \mu \mathrm{m}$. b, A vessel of the medial frontal gyrus is surrounded by activated perivascular macrophages. Scale bar, $20 \mu \mathrm{m}$. c, The cortical surface is shown. The upper third of the figure contains the leptomeninges that cover the cortex. A dense b Perivascular macrophage activation

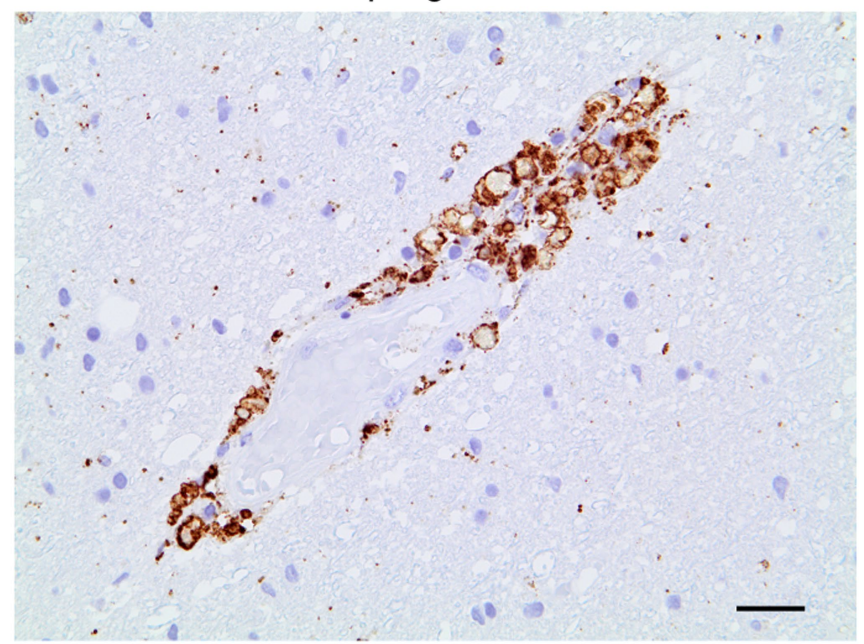

d Summary of immune activation in situ in COVID-19

\begin{tabular}{|c|c|c|c|}
\hline Patient & $\begin{array}{c}\text { Meningeal } \\
\text { Macrophage } \\
\text { infiltration }\end{array}$ & $\begin{array}{c}\text { Cortical } \\
\text { Microglia } \\
\text { content }\end{array}$ & $\begin{array}{c}\text { White } \\
\text { matter } \\
\text { Microglia } \\
\text { content }\end{array}$ \\
\hline 1 & moderate & mild & severe \\
\hline 2 & severe & severe & excessive \\
\hline 3 & - & severe & excessive \\
\hline 4 & mild & moderate & severe \\
\hline 5 & mild & mild & severe \\
\hline 6 & mild & moderate & severe \\
\hline 7 & moderate & mild & moderate \\
\hline 8 & moderate & moderate & moderate \\
\hline
\end{tabular}

infiltration by brown stained macrophages into the leptomeninges is visible. Scale bar, $20 \mu \mathrm{m}$.d, Summary of innate immune reactivity across eight patients with COVID-19, typically not observed in healthy brains at these levels, colour-coded and labelled by severity. A semiquantitative categorization for changes, as usual in the field of pathology, is used: mild = detectable microgliosis, atypical for healthy tissue; moderate $=$ a pathological process typical of pathological changes; severe $=$ a marked pathological process. Several clusters of microglia or macrophages were characterized as excessive beyond the severe category. Immunohistochemical stains are representative of at least two independent experiments. 
a COVID inflamed/ activated astrocyte cluster

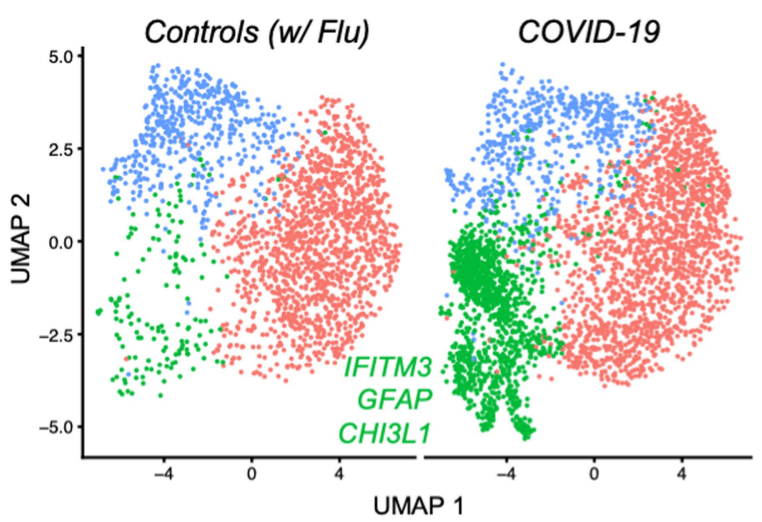

b COVID astrocyte freq.

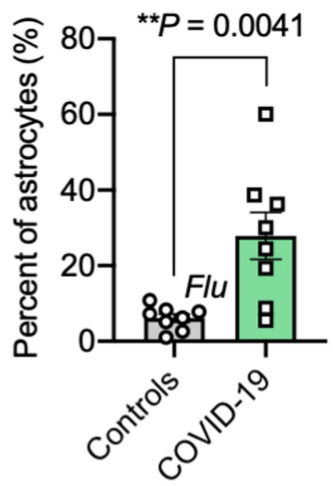

d

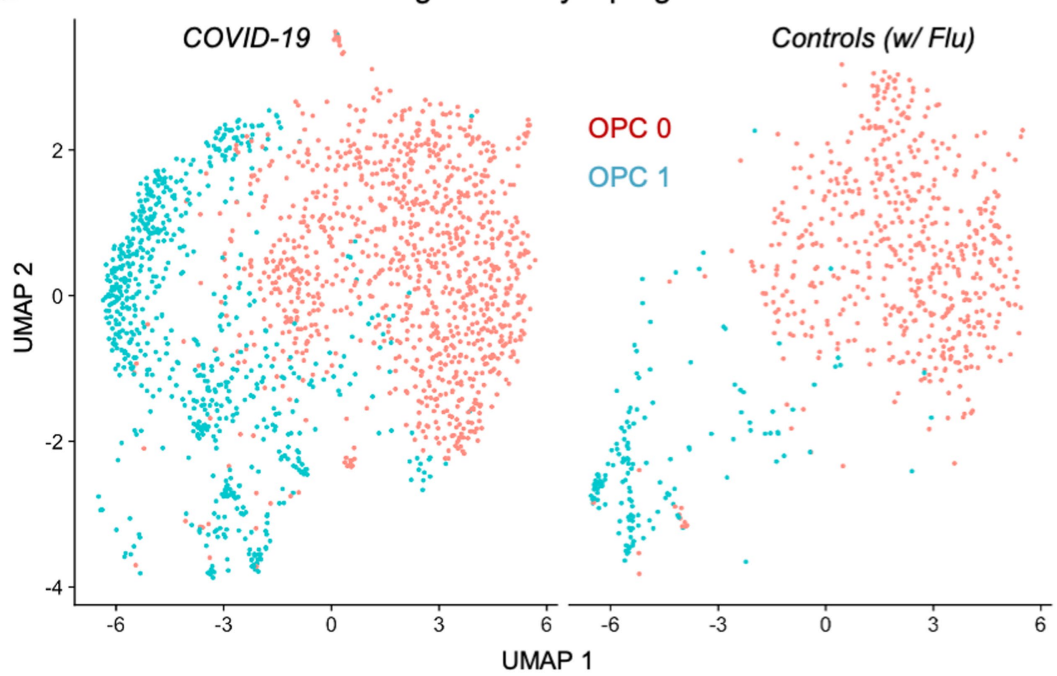

f

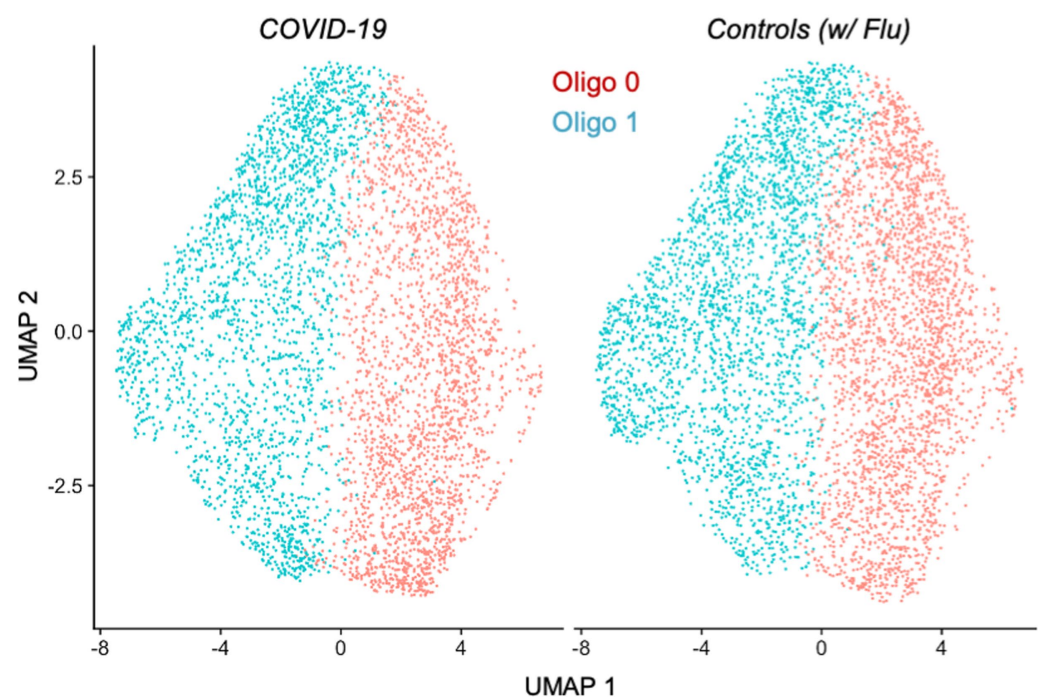

c Dysregulated COVID astrocyte pathways

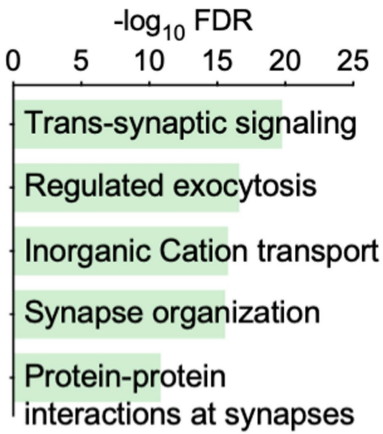

e $\quad$ OPC 1 frequency

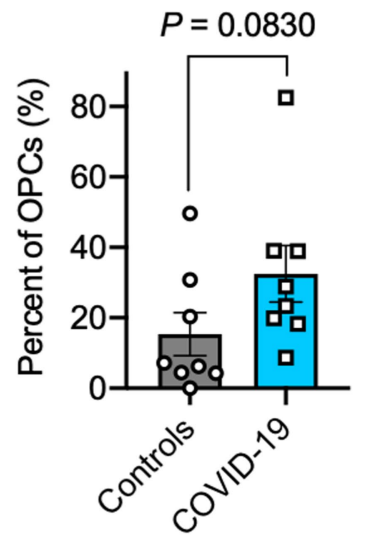

g Oligo 1 frequency (no enrichment)

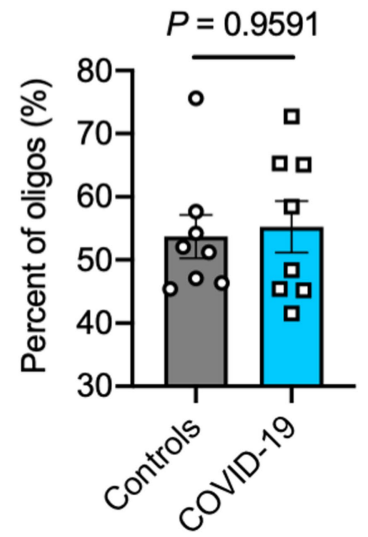

Extended Data Fig. 12 |See next page for caption. 


\section{Article}

Extended Data Fig. 12 | Evaluation of COVID-19-enriched subpopulations in other parenchymal glia. a, UMAP of astrocytes captured in the human frontal cortex, split by control individuals (including influenza, $n=8$ ) and patients with COVID-19 $(n=8)$. Cells are coloured by cell-type subcluster. Genes upregulated in the COVID-19-enriched astrocyte cluster are labelled in green. b, Quantification of astrocyte cluster 1 as a proportion of total astrocytes $(n=8$ control, including influenza; $n=8$ COVID-19, two-sided Mann-Whitney $t$-test $P=0.0041$; mean \pm s.e.m.). Example genes upregulated in the COVID-19associated astrocyte cluster are shown. c, Enriched biological pathways $\left(\right.$ Metascape $\left.^{54}\right)$ amongst upregulated gene markers of COVID-19 astrocytes. Enrichment is based on FDR-corrected cumulative hypergeometric $P$ values (Bonferroni correction FDR < 0.05; MAST with default thresholds). d, UMAP projection of OPCs and trending but not significant emergence of a COVID-19enriched subcluster. e, Quantification of the frequency of the COVID-19enriched OPC subcluster as a proportion of all OPCs $(n=8$ control, including 1 influenza and $n=8$ COVID-19, two-sided Mann-Whitney $t$-test, $P=0.083$; mean \pm s.e.m., not significant).f, $\mathbf{g}$, As in d, e, respectively, but for mature oligodendrocytes with $P=0.9591$. 


\section{Reporting Summary}

Nature Research wishes to improve the reproducibility of the work that we publish. This form provides structure for consistency and transparency in reporting. For further information on Nature Research policies, see our Editorial Policies and the Editorial Policy Checklist.

\section{Statistics}

For all statistical analyses, confirm that the following items are present in the figure legend, table legend, main text, or Methods section.

n/a $\mid$ Confirmed

$\bigotimes$ The exact sample size $(n)$ for each experimental group/condition, given as a discrete number and unit of measurement

$\square$ A statement on whether measurements were taken from distinct samples or whether the same sample was measured repeatedly

The statistical test(s) used AND whether they are one- or two-sided

Only common tests should be described solely by name; describe more complex techniques in the Methods section.

$\bigotimes$ A description of all covariates tested

$\bigotimes$ A description of any assumptions or corrections, such as tests of normality and adjustment for multiple comparisons

$\triangle$ A full description of the statistical parameters including central tendency (e.g. means) or other basic estimates (e.g. regression coefficient)

AND variation (e.g. standard deviation) or associated estimates of uncertainty (e.g. confidence intervals)

For null hypothesis testing, the test statistic (e.g. $F, t, r$ ) with confidence intervals, effect sizes, degrees of freedom and $P$ value noted Give $P$ values as exact values whenever suitable.

Х $\square$ For Bayesian analysis, information on the choice of priors and Markov chain Monte Carlo settings

Х $\square$ For hierarchical and complex designs, identification of the appropriate level for tests and full reporting of outcomes

$\triangle \square$ Estimates of effect sizes (e.g. Cohen's $d$, Pearson's $r$ ), indicating how they were calculated

Our web collection on statistics for biologists contains articles on many of the points above.

\section{Software and code}

Policy information about availability of computer code

Data collection For droplet-based snRNA-seq, libraries were prepared using the Chromium Next GEM Single Cell $3^{\prime}$ v3.1 according to the manufacturer's protocol (10x Genomics), targeting 10,000 nuclei per sample after counting with a TC20 Automated Cell Counter (Bio-Rad). Generated snRNAseq libraries were sequenced across $\$ 4$ lanes on a NovaSeq 6000 (150 cycles, Novogene).

Data analysis Gene counts were obtained by aligning reads to the hg38 genome (refdata-gex-GRCh38-2020-A) using CellRanger software (v.4.0.0) (10x Genomics). Data were primarily analyzed using the Seurat 3.2.1 package in R (v.3.6.3). Specific analysis also used the packages: Monocle3, CellChat (v.0.02), Enrichr, Metascape, GeneTrail 3, dplyr, dplyr (v.1.0.0), ggplot2 (v.3.2.2.), patchwork (v.1.0.1), openxlsx (v.4.1.5), bioconductor-scater (v.1.14.6), bioconductor-dropletutils (v1.6.1), bioconductor-complexheatmap (v.2.2.0), tidyverse (v.1.3.0), and Isa (v.0.73.2). All other tasks were performed on an x86_64-based Ubuntu (4.15.0-55-generic kernel) server cluster.

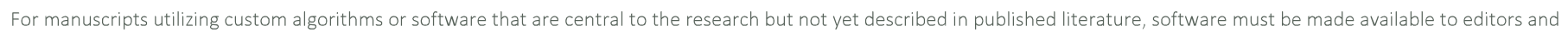

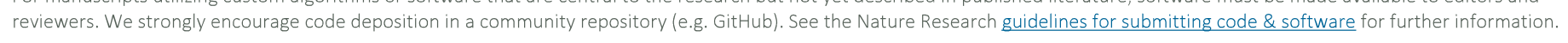

\section{Data}

Policy information about availability of data

All manuscripts must include a data availability statement. This statement should provide the following information, where applicable:

- Accession codes, unique identifiers, or web links for publicly available datasets

- A list of figures that have associated raw data

- A description of any restrictions on data availability

Raw sequencing data has been deposited in NCBI GEO: GSE159812. Normalized counts data are also available for download at: https://twc-stanford.shinyapps.io/scRNA_Brain_COVID19. 


\section{Field-specific reporting}

Please select the one below that is the best fit for your research. If you are not sure, read the appropriate sections before making your selection.

\ Life sciences $\quad \square$ Behavioural \& social sciences $\quad \square$ Ecological, evolutionary \& environmental sciences

For a reference copy of the document with all sections, see nature.com/documents/nr-reporting-summary-flat.pdf

\section{Life sciences study design}

All studies must disclose on these points even when the disclosure is negative.

Sample size We did not use statistical methods to pre-determine sample sizes, but our sample sizes are similar to those reported in previous publications. Sample size was primarily determined by the availability of high-quality tissue that was dissected consistently for brain region.

Data exclusions Low-quality nuclei were excluded using standard approaches: (1) ambient cell free mRNA contamination was removed using SoupX for each individual sample; (2) outliers with a high ratio of mitochondrial ( $>5 \%,<200$ features) relative to endogenous RNAs and homotypic doublets (> 5000 features) were removed in Seurat; and (3) after scTransform normalization and integration, doublets and multiplets were filtered out using DoubletFinder with subsequent manual inspection and filtering based on cell type-specific marker genes.

Replication Though there is limited availability of high-quality COVID-19 fresh-frozen postmortem brain tissue, we have since doubled our sample sizes and found that our original results hold.

Randomization Not applicable, cannot decide whether a patient is infected and suffers from COVID-19.

Blinding Experimenter was blinded to samples during tissue processing and nuclei isolation steps.

\section{Reporting for specific materials, systems and methods}

We require information from authors about some types of materials, experimental systems and methods used in many studies. Here, indicate whether each material, system or method listed is relevant to your study. If you are not sure if a list item applies to your research, read the appropriate section before selecting a response.

Materials \& experimental systems

$\mathrm{n} / \mathrm{a}$ Involved in the study

$\square$ Antibodies

Х $\square$ Eukaryotic cell lines

\ $\square$ Palaeontology and archaeology

Methods

\ $\square$ Animals and other organisms

$\bigotimes \square$ Human research participants

Х Clinical data

Х $\square$ Dual use research of concern

\section{Antibodies}

Antibodies used

SARS spike glycoprotein antibody 3A2 (rabbit, Abcam ab272420, 1:100), SARS-CoV2 spike antibody (mouse, GeneTex GTX632604, 1 A9 clone, 1:100), SARS-CoV2 spike antibody (rabbit, Sino Biological 40150-T62-CoV2, 1:100), SARS-CoV2 Nucleoprotein antibody (rabbit, Sino Biological 40143-T62, 1:100), and CD68 (mouse, Dako M0876, PG-M1 clone, 1:100).

Validation

Antibodies were chosen because of their validation in prior publications. 